\title{
Isolas of 2-pulse solutions in homoclinic snaking scenarios
}

\author{
Jürgen Knobloch \\ Department of Mathematics \\ Technical University Ilmenau \\ 98684 Ilmenau, Germany \\ Björn Sandstede \\ Division of Applied Mathematics \\ Brown University \\ Providence, RI 02912, USA
}

\author{
David J.B. Lloyd \\ Department of Mathematics \\ University of Surrey \\ Guildford, GU2 7XH, UK \\ Thomas Wagenknecht \\ Department of Applied Mathematics \\ University of Leeds \\ Leeds, LS2 9JT, UK
}

October 27, 2010

\begin{abstract}
Homoclinic snaking refers to the bifurcation structure of symmetric localised roll patterns that are often found to lie on two sinusoidal "snaking" bifurcation curves, which are connected by an infinite number of "rung" segments along which asymmetric localised rolls of various widths exist. The envelopes of all these structures have a unique maximum and we refer to them as symmetric or asymmetric 1-pulses. In this paper, the existence of stationary 1D patterns of symmetric 2-pulses that consist of two well-separated 1-pulses is established. Corroborating earlier numerical evidence, it is shown that symmetric 2-pulses exist along isolas in parameter space that are formed by parts of the snaking curves and the rungs mentioned above.
\end{abstract}

\section{Introduction}

The emergence of localised patterns in bistable systems has attracted much attention in recent years with particular emphasis being given to the phenomenon known as homoclinic snaking. This phenomenon refers to the appearance and disappearance of infinitely many stationary localised patterns through virtually simultaneous saddle-node bifurcations and, more specifically, to the property that all these emerging patterns are globally connected in parameter space. In particular, there is an open region in parameter space in which infinitely many localised patterns coexist. The interior part of these patterns resembles a spatially periodic structure, so that one can interpret them as periodic waves that are subjected to a localised amplitude modulation. As one follows these patterns in the bifurcation parameter, their interior domain broadens indefinitely.

In this paper, we focus on one-dimensional patterns. As an example, consider the Swift-Hohenberg equation

$$
U_{t}=-\left(1+\partial_{x}^{2}\right)^{2} U+\mu U+\nu U^{2}-U^{3}, \quad x \in \mathbb{R}, \quad U \in \mathbb{R}
$$

with $\nu=2$. Stationary localised patterns of the Swift-Hohenberg equation with $\nu=2$ can be computed as homoclinic orbits of the steady-state equation

$$
\left(1+\partial_{x}^{2}\right)^{2} U-\mu U-2 U^{2}+U^{3}=0
$$

or, equivalently, of the associated first-order system

$$
u_{x}=f(u, \mu), \quad u=\left(u_{1}, u_{2}, u_{3}, u_{4}\right)^{T}=\left(U, U_{x}, U_{x x}, U_{x x x}\right)^{T} \in \mathbb{R}^{4}
$$



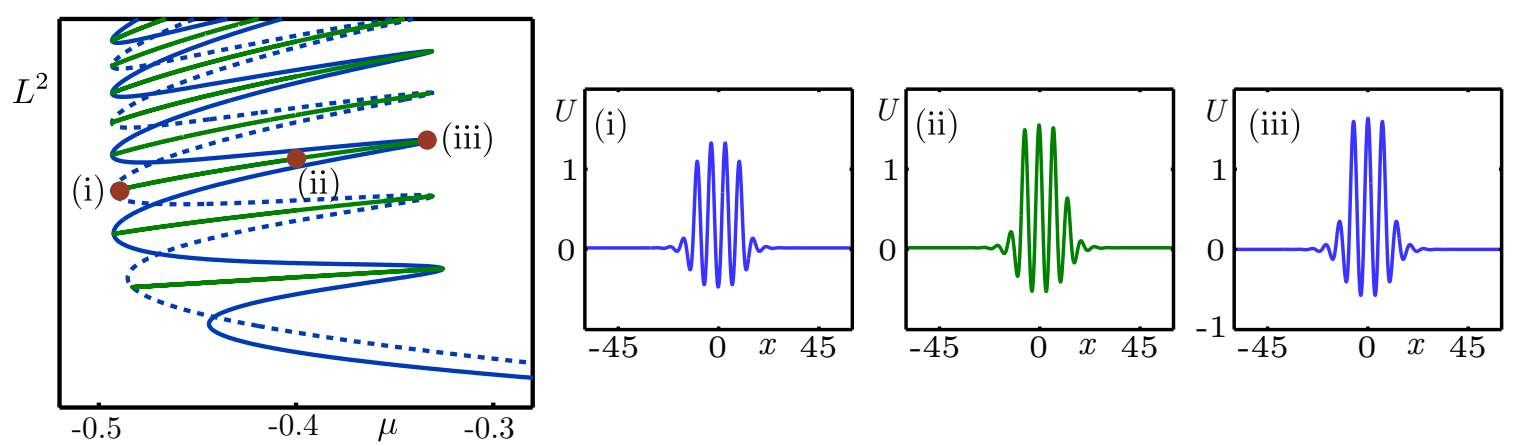

Figure 1: In the left panel, the $L^{2}$-norm of 1-homoclinic orbits of the Swift-Hohenberg equation (1.1) is plotted against the parameter $\mu$. The three panels to the right show the solution profiles $U(x)$ as functions of $x$ along one of the rungs that connect the two snaking curves that are plotted as solid and dashed curves.

with

$$
f(u, \mu)=\left(u_{2}, u_{3}, u_{4},-2 u_{3}+(\mu-1) u_{1}+2 u_{1}^{2}-u_{1}^{3}\right)^{T} .
$$

Homoclinic snaking in (1.1) has been discussed in a number of papers, and we refer to $[2,4]$ for a comprehensive list of references and to $[11,16]$ for two recent reviews that also list numerous applications in the natural sciences. The characteristic bifurcation diagram for 1-homoclinic orbits to the origin is shown in Figure 1. It consists of two intertwined snaking curves corresponding to homoclinic orbits of (1.1) that are symmetric under the reflection $x \mapsto-x$ : as shown in the rightmost panels in Figure 1, the symmetric orbits along the two different snaking curves differ by whether they are symmetric about a maximum or minimum. The two snaking curves are connected by rungs or ladders that correspond to non-symmetric homoclinic orbits. We refer to the union of the two snaking curves and the rung branches as the double-helix structure.

The geometric foundation of homoclinic snaking is well understood. The homoclinic orbits bifurcate from a heteroclinic cycle that is formed by connecting orbits between the equilibrium $U=0$ and a periodic orbit. A geometric explanation of snaking, based on a careful inspection of the intersections of the stable and unstable manifolds of the underlying equilibrium and periodic orbits, has been given in [25]; see also [10]. For the special case of the Swift-Hohenberg equation, this picture has been put on a firm footing in $[9,18]$ using normal-form theory and beyond-all-orders asymptotics. On a general level, the precise link between the global geometry of the heteroclinic connections and the resulting snaking diagrams of both symmetric and non-symmetric solutions has been elucidated in [2]. From a structural viewpoint, the key ingredients necessary for snaking to occur are reversibility (that is, the invariance under reflection in $x$ ) and, to some extent, the property that the underlying equation is conservative or Hamiltonian. If the system is not conservative, then snaking may still occur but the non-symmetric solutions will, in general, correspond to travelling waves and not to stationary states.

The papers mentioned above focused on 1-homoclinic orbits, that is, on homoclinic solutions that follow the underlying heteroclinic cycle precisely once. The general bifurcation results by Devaney [12] or Härterich [13] imply that each of the 1-homoclinic orbits described above will, for each fixed parameter value, be accompanied by families of $N$-homoclinic solutions that follow the heteroclinic cycle precisely $N$ times provided the underlying equilibrium is a bi-focus. The results in $[12,13]$ also indicate that, for each $N$, there will be infinitely many $N$-homoclinic orbits that differ by the separation distances between consecutive pulses. Our goal is to analyse their existence in homoclinic snaking scenarios, where the 1-homoclinic orbits arise in global bifurcations from heteroclinic cycles. We shall focus on symmetric 2-homoclinic orbits.

Multi-pulse solutions in snaking scenarios were first studied numerically in [14, 24], where it was observed that 2pulse solutions lie on closed curves near the original snaking curves. Various different types of isolas that differed by the number of fold bifurcations they contained were found in these two papers. To gain further insight into 2-pulse solutions, we computed symmetric 2-homoclinic orbits for the Swift-Hohenberg equation (1.1) using 

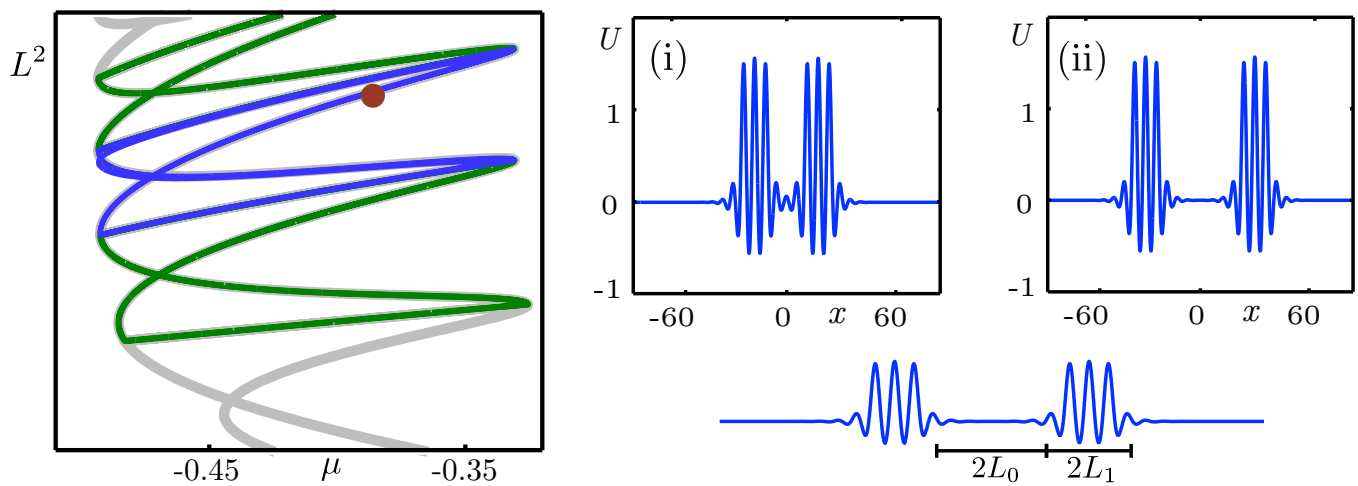

Figure 2: Plotted are numerically computed figure-eight isolas associated with 2-homoclinic orbits of the SwiftHohenberg-equation (1.1). If we fix the separation distance $2 L_{0}$ between the two pulses, we find a family of stacked isolas whose solution profiles differ by the width $2 L_{1}$ of each individual pulse. If we fix the width of each pulse, we find a family of nested isolas whose profiles differ by the separation distance between the two pulses as illustrated by the profiles in panels (i)-(ii) that lie on two different isolas near the bullet in the left panel.
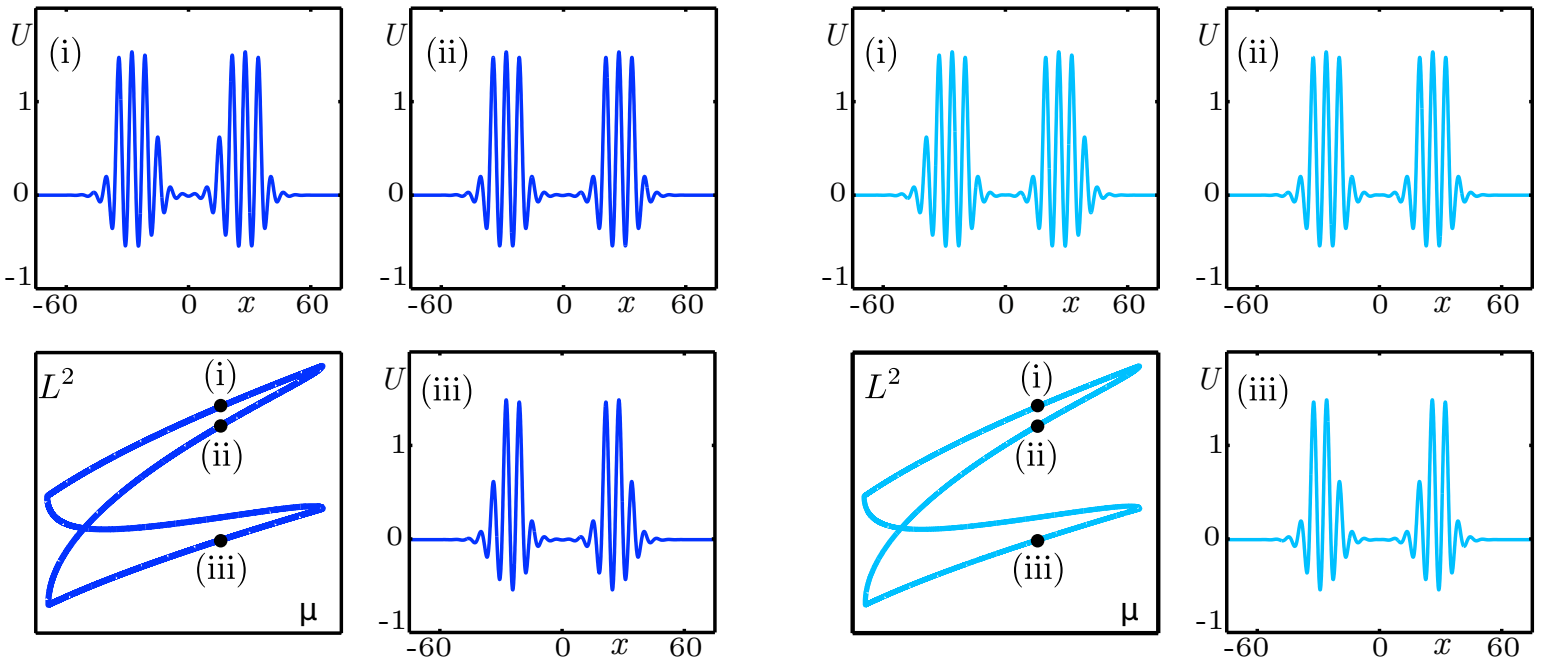

Figure 3: Shown are numerical computations of two different types of 2-pulse solutions that differ by whether their central point is a positive maximum or negative minimum (left panels) or a negative maximum or positive minimum (right panels).

the homoclinic branch-switching method developed in [21] and show the resulting bifurcation diagram and the associated solution profiles in Figure 2. This diagram shows the double helix of symmetric and non-symmetric 1 -pulses in grey and a number of closed bifurcation curves $^{1}$ of symmetric 2 -pulses in green and blue. We can see that the branches of 2-pulses follow the double helix very closely but that all bifurcation curves belonging to 2-pulses are isolas of figure-eight type. As indicated in Figure 2, we found a family of stacked isolas that differ by the widths of each of the pulses that form the associated 2-pulse profiles. If we fix the width of each pulse, then we found a family of nested isolas that are parametrized by a discrete set of increasing separation distances between the two pulses. We often encountered isolas that initially appeared to contain more fold bifurcations than present in a single figure-eight isola, but these isolas were found to break up into several figure-eight isolas once the step size of the continuation scheme was decreased sufficiently. This observation was also made independently in the recent work [6].

One can think of 2-pulses as being composed of two pulses that both mirror the behaviour of the symmetric and

\footnotetext{
${ }^{1}$ Plotted are the $L^{2}$-norm of 1-pulses and half the $L^{2}$-norm of 2-pulses to make a comparison meaningful.
} 
non-symmetric pulses along the parts of the original double helix that are closest to the isola. It is interesting to note that, along each rung, there are two non-symmetric 1-pulses that are related by symmetry. In particular, we can create two different symmetric 2-pulses along the rungs by forming a 2-pulse from each of the two nonsymmetric 1-pulses. This is further illustrated in Figure 3. As shown there, we found two different types of 2-pulses that can be characterised as follows. In the center $x=0$ of each 2-pulse with profile $U(x)$, we have $U_{x}(0)=U_{x x x}(0)=0$. If $x=0$ corresponds to a positive maximum or a negative minimum of the profile, then the solution behaves as shown in the left panels in Figure 3. On the other hand, the behaviour will be as shown in the right panels in Figure 3 if the center $x=0$ is a negative maximum or a positive minimum of the solution profile. The existence of these different types of 2-homoclinic orbits is a typical phenomenon, as the analysis in [8] shows in a slightly different context.

In this paper, we will refine these results and put them on an analytic footing. We will show that the isolas of figure-eight type are the only possible bifurcation curves for symmetric 2-homoclinic orbits provided that the width of the pulses is sufficiently large and that they are sufficiently well separated. Our analysis follows the approach in [2]. In $\S 2$, we present our assumptions, which are slightly more restrictive than those in [2], and our main results. The existence analysis of 2 -pulses is then carried out in $\S 3$. In $\S 4$, we give a geometric interpretation of our results and outline generalizations to non-symmetric 2-pulses and, more generally, to $N$-pulses.

\section{Existence results for 2-pulses}

We consider four-dimensional systems

$$
u_{x}=f(u, \mu), \quad u \in \mathbb{R}^{4}, \quad \mu \in \mathbb{R},
$$

where $f$ will be a smooth vector field. We shall assume that (2.1) possesses a heteroclinic cycle between the equilibrium $u=0$ and a periodic orbit, and are interested in locating homoclinic orbits near this cycle.

Firstly, however, let us specify the general properties of the vector field $f$. We require that $(2.1)$ is both reversible and Hamiltonian. More precisely, we assume the following.

Hypothesis (H1) There exists a linear map $\mathcal{R}: \mathbb{R}^{4} \rightarrow \mathbb{R}^{4}$ with $\mathcal{R}^{2}=1$ and $\operatorname{dim} \operatorname{Fix}(\mathcal{R})=2$ so that $f(\mathcal{R} u, \mu)=$ $-\mathcal{R} f(u, \mu)$ for all $(u, \mu)$.

Reversibility as encoded in Hypothesis (H1) implies that $\mathcal{R} u(-x)$ satisfies (2.1) whenever $u(x)$ does. Solutions with $u(0) \in \operatorname{Fix}(\mathcal{R}):=\left\{u \in \mathbb{R}^{4}: \mathcal{R} u=u\right\}$ are said to be symmetric as they satisfy $u(x)=\mathcal{R} u(-x)$ for all $x$.

Hypothesis (H2) There exists a smooth function $\mathcal{H}: \mathbb{R}^{4} \times \mathbb{R} \rightarrow \mathbb{R}$ with $\mathcal{H}(\mathcal{R} u, \mu)=\mathcal{H}(u, \mu)$, such that

$$
f(u, \mu)=\mathcal{J H}_{u}(u, \mu), \quad \mathcal{J}=\left(\begin{array}{cc}
0 & -1 \\
1 & 0
\end{array}\right),
$$

and $\mathcal{H}(0, \mu)=0$ for all $\mu$.

Next, we introduce the solutions of (2.1) that we will consider. We shall assume that there is a compact interval $J \subset \mathbb{R}$ with nonempty interior $\stackrel{\circ}{J}$ such that the following hypotheses are met for $\mu \in J$.

Hypothesis (H3) The origin $u=0$ is a saddle-focus of (2.1) for all $\mu \in J$, that is, the linearised vector field has a quadruplet of complex conjugate eigenvalues so that there is a constant $\kappa>0$ with

$$
\operatorname{spec}\left(f_{u}(0, \mu)\right)=\left\{ \pm \alpha_{0}(\mu) \pm \mathrm{i} \beta_{0}(\mu)\right\}
$$

and $\alpha_{0}(\mu), \beta_{0}(\mu) \geq \kappa>0$ for all $\mu \in J$. 
Hypothesis (H4) We assume that (2.1) has, for each $\mu \in J$, a periodic orbit $\gamma(x, \mu)$ with minimal period $2 \pi$ which satisfy the following for each $\mu \in J$ :

(i) The family $\gamma(x, \mu)$ depends smoothly on $\mu$.

(ii) $\gamma(x, \mu)$ is symmetric with $\gamma(0, \mu) \in \operatorname{Fix}(\mathcal{R})$.

(iii) $\mathcal{H}(\gamma(x, \mu), \mu)=0$ and $\mathcal{H}_{u}(\gamma(x, \mu), \mu) \neq 0$ for all $x \in \mathbb{R}$.

(iv) $\gamma(x, \mu)$ has two positive Floquet multipliers $\mathrm{e}^{ \pm 2 \alpha_{1}(\mu)}$ with $\alpha_{1}(\mu) \geq \kappa>0$ uniformly in $\mu \in J$.

Reversibility implies that both the spectrum of $u=0$ and the set of characteristic exponents of $\gamma(x, \mu)$ are symmetric under multiplication by -1 . Furthermore, the Hamiltonian property of (2.1) allows us to restrict the search for connecting orbits to the zero level set of $\mathcal{H}$.

The above setting fits into the context of [2]. Indeed, Hypotheses (H2) and (H3) are more restrictive than the corresponding assumptions made in [2], where we assumed only that $f$ has a first integral and that the equilibrium $u=0$ is hyperbolic without assuming anything else about its eigenvalues. We now recall the following result from [2].

Lemma 2.1 ([2]) Assume that Hypotheses (H1)-(H4) are met, then there exist a $\delta>0$, a smooth reversible change of coordinates near $\gamma(\cdot, \mu)$, and smooth real-valued functions $h^{c}, h_{j}^{s}$ and $h_{j}^{u}$ with $j=1,2$ so that (2.1) restricted to the zero level set of $\mathcal{H}$ is, for all $\mu \in J$, of the form

$$
\begin{aligned}
v_{x}^{c} & =1+h^{c}(v, \mu) v^{s} v^{u} \\
v_{x}^{s} & =-\left[\alpha_{1}(\mu)+h_{1}^{s}(v, \mu) v^{s}+h_{2}^{s}(v, \mu) v^{u}\right] v^{s} \\
v_{x}^{u} & =\left[\alpha_{1}(\mu)+h_{1}^{u}(v, \mu) v^{s}+h_{2}^{u}(v, \mu) v^{u}\right] v^{u},
\end{aligned}
$$

where $v=\left(v^{c}, v^{s}, v^{u}\right) \in S^{1} \times I \times I$ with $I=[-\delta, \delta]$. The reverser $\mathcal{R}$ acts on $v$ via $\mathcal{R}\left(v^{c}, v^{s}, v^{u}\right)=\left(-v^{c}, v^{u}, v^{s}\right)$.

The dynamics we are interested in is organised by heteroclinic cycles that connect the equilibrium $u=0$ and the periodic orbit $\gamma$. The underlying heteroclinic orbits correspond to intersections of the stable manifold $W^{s}(0, \mu)$ of the equilibrium $u=0$ and the strong unstable fibres $W^{u u}(\gamma(\varphi, \mu), \mu)$ of the periodic orbit $\gamma$. To capture these intersections, we define the sections

$$
\Sigma_{1}^{\text {in }}:=S^{1} \times\left\{v^{s}=\delta\right\} \times I, \quad \Sigma_{1}^{\text {out }}:=S^{1} \times I \times\left\{v^{u}=\delta\right\}
$$

in the Fenichel coordinates of Lemma 2.1 near the periodic orbit $\gamma(\cdot, \mu)$ in the zero level set of $\mathcal{H}$ and set

$$
\Gamma:=\left\{(\varphi, \mu) \in S^{1} \times J: W^{s}(0, \mu) \cap W^{u u}(\gamma(\varphi, \mu), \mu) \cap \Sigma_{1}^{\text {out }} \neq \emptyset\right\} .
$$

We now state two hypotheses that encapsulate the geometric assumptions we impose on the set $\Gamma$ and the various invariant manifolds introduced above. These hypotheses are illustrated in Figures 4-5 and will be motivated in more detail below. First, we assume that $\Gamma$ is the graph of a function that maps $S^{1}$ into $\stackrel{\circ}{J}$.

Hypothesis (H5) The set $\Gamma$ is the graph of a smooth function $z: S^{1} \rightarrow \stackrel{\circ}{J}$. Furthermore, we assume that $z^{\prime}(\varphi)=0$ if and only if $\varphi \in\left\{\ell_{\mathrm{m}}, \ell_{\mathrm{M}}\right\}$ and that $z^{\prime \prime}\left(\ell_{\mathrm{m}, \mathrm{M}}\right) \neq 0$. In other words, $z$ has precisely two critical points, namely a minimum and a maximum, which are both nondegenerate.

Thus, we assume that the stable manifold $W^{s}(0, \mu)$ intersects the strong unstable fiber of $\gamma(\varphi)$ in the section $\Sigma_{1}^{\text {out }}$ if, and only if, $\mu=z(\varphi)$. Hypothesis (H5) also assumes that the function $z(\varphi)$ has the shape shown in Figure 5. Specifically, heteroclinic orbits between $u=0$ and the periodic orbit $\gamma$ are created and destroyed in two saddle-node bifurcations that occur when $\mu=z(\varphi)$ with $\varphi=\ell_{\mathrm{m}, \mathrm{M}}$. Hypothesis (H5) can be relaxed considerably, and we will discuss this in detail in $\S 4$. 

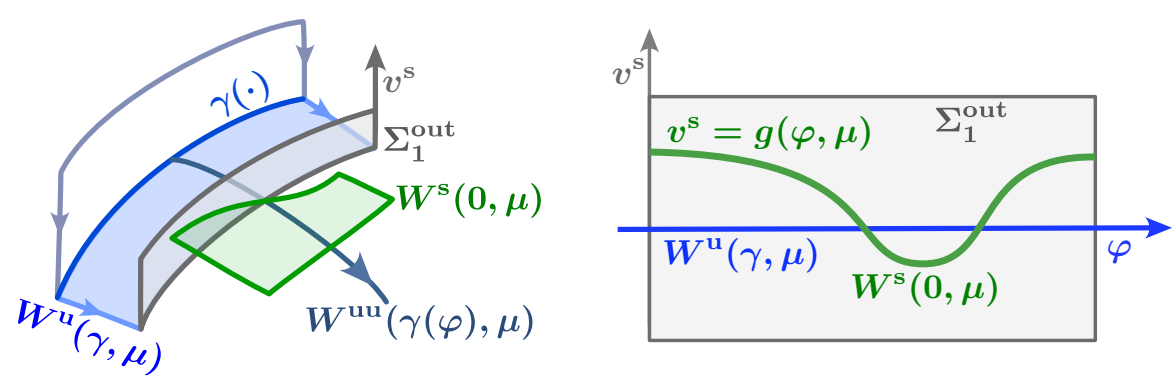

Figure 4: The geometry of the stable manifold $W^{\mathrm{s}}(0, \mu)$, the unstable manifold $W^{\mathrm{u}}(\gamma, \mu)$ and the strong unstable fibers $W^{\mathrm{uu}}(\gamma(\varphi), \mu)$ that we assumed in Hypotheses (H5)-(H6) is illustrated.
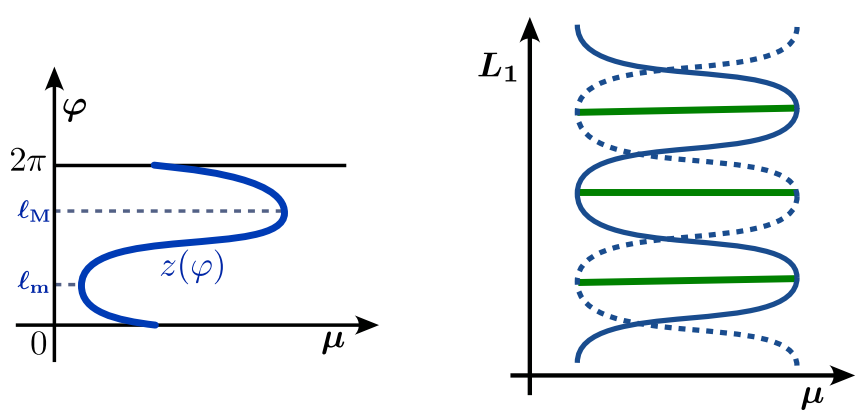

Figure 5: Shown are the function $\mu=z(\varphi)$ and the snaking diagram of symmetric 1-homoclinic orbits.

Hypothesis (H6) There exist a constant $\eta>0$ and a smooth function $g: S^{1} \times J \rightarrow I$ so that

$$
W^{s}(0, \mu) \cap \Sigma_{1}^{\text {out }}=\left\{\left(v^{c}, v^{s}, v^{u}\right)=(\varphi, g(\varphi, \mu), \delta): \varphi \in S^{1}\right\}
$$

for each $\mu \in J$ and $\left|g_{\mu}(\varphi, \mu)\right| \geq \eta>0$ for all $(\varphi, \mu)$.

Hypothesis (H6) makes two different assumptions. First, we assume that the parameter $\mu$ moves the stable manifold $W^{s}(0, \mu)$ up and down in a monotonic fashion relative to the unstable manifold $W^{u}(\gamma(\cdot, \mu), \mu)$ of the periodic orbit. This assumption is satisfied, for instance, for near-integrable systems such as the normal form of a Hamiltonian-Hopf bifurcation, which corresponds to a Turing bifurcation of the underlying partial differential equations; see [25] and the references therein. The second assumption in Hypothesis (H6) is that $W^{s}(0, \mu)$ is a graph over $W^{u}(\gamma(\cdot, \mu), \mu)$ inside the section $\Sigma_{1}^{\text {out }}$. In particular, we assume that the manifold $W^{s}(0, \mu)$ intersects the section $\Sigma_{1}^{\text {out }}$ along a circle for all $\mu \in J$. This assumption can be relaxed, and we refer to $\S 4$ for details.

Note that the definition of $\Gamma$ in (2.3) and Hypotheses (H5)-(H6) imply that

$$
g(\varphi, \mu)=0 \quad \Longleftrightarrow \quad \mu=z(\varphi) .
$$

If Hypotheses (H1)-(H6) are met, then (2.1) exhibits homoclinic snaking. More precisely, it was shown in [2] that there are constants $\kappa>0, L_{*} \gg 1$, and functions $\mu_{*}\left(L_{1}, \varphi\right)$ and $Z_{0}\left(L_{1}, \varphi\right)$ with

$$
\mu_{*}\left(L_{1}, \varphi\right)=z\left(L_{1}+\varphi\right)+\mathrm{O}\left(\mathrm{e}^{-\kappa L_{1}}\right), \quad Z_{0}\left(L_{1}, \varphi\right)=z\left(L_{1}+\varphi\right)-z\left(L_{1}-\varphi\right)+\mathrm{O}\left(\mathrm{e}^{-\kappa L_{1}}\right), \quad L \geq L_{*}
$$

and $Z_{0}\left(L_{1},-\varphi\right)=-Z_{0}\left(L_{1}, \varphi\right)$ such that the following holds. Equation (2.1) has a symmetric 1-homoclinic orbit to the origin that spends time $2 L_{1}$ near the periodic orbit precisely when $\mu=\mu_{*}\left(L_{1}, 0\right)$ or $\mu=\mu_{*}\left(L_{1}, \pi\right)$; see Figure 5 for an illustration. Furthermore, non-symmetric 1-homoclinic orbits exist precisely when $\mu=\mu_{*}\left(L_{1}, \varphi\right)$ and $Z_{0}\left(L_{1}, \varphi\right)=0$, and it is not difficult to see that this gives the horizontal rungs curves that connect the two snaking curves shown in Figure 5; we refer to [2] for further details. In particular, given that we know the function $\mu_{*}\left(L_{1}, \varphi\right)$, the information about both symmetric and non-symmetric 1-homoclinic orbits is contained 

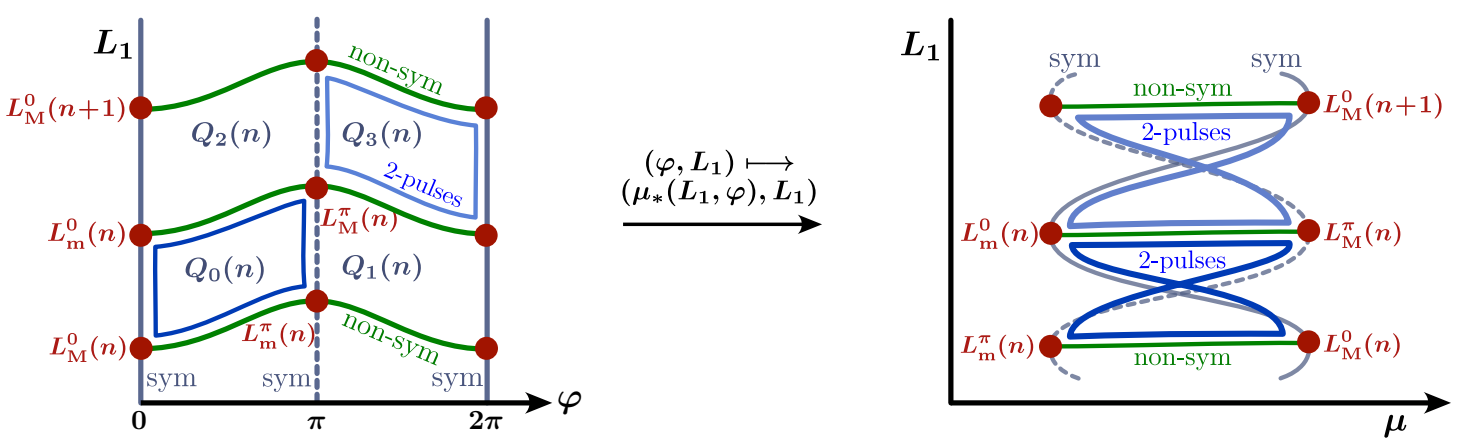

Figure 6: The bifurcation curves of symmetric (labelled sym) and non-symmetric (labelled non-sym) 1-homoclinic orbits are shown in the $\left(\varphi, L_{1}\right)$ and the $\left(\mu, L_{1}\right)$-planes. Non-symmetric 1-pulses bifurcate from symmetric 1-pulses at the pitchfork bifurcation points shown as bullets. Symmetric 2-homoclinic orbits exist on isolas near the original snaking curves.

in the function $Z_{0}\left(L_{1}, \varphi\right)$ or, more precisely, in its zero level set $Z_{0}^{-1}$ in the $\left(\varphi, L_{1}\right)$-plane. This is illustrated further in Figure 6, which contains the vertical lines $\varphi=0$ and $\varphi=\pi$ in $Z_{0}^{-1}$ as well as the connecting rungs curves corresponding to non-symmetric 1-pulses that emerge via pitchfork bifurcations from symmetric 1-pulses at the discrete set

$$
L_{1}=L_{i}^{\varphi}(n)=\ell_{i}+\varphi+2 \pi n+\mathrm{O}\left(\mathrm{e}^{-\kappa n}\right), \quad i=\mathrm{m}, \mathrm{M}, \quad \varphi \in\{0, \pi\}, \quad n \geq n_{*}
$$

of bifurcation points. Recall here that $\ell_{\mathrm{m}}$ and $\ell_{\mathrm{M}}$ correspond, respectively, to the minimum and maximum of the function $z(\varphi)$. For each fixed $n$, the set $Z_{0}^{-1}$ encloses precisely four open rectangular sets that we denote by $Q_{j}(n)$ with $j=0, \ldots, 3$. We can now state our result on the existence of 2-homoclinic orbits.

Theorem 1 Assume that Hypotheses (H1)-(H6) are met. There exist integers $m_{*}, n_{*} \gg 1$ such that, for each pair $(m, n)$ of integers with $m \geq m_{*}$ and $n \geq n_{*}$, there are precisely two closed curves without self-intersection inside each rectangular set $Q_{j}(n)$ with $j=0, \ldots, 3$ along which 2-homoclinic orbits with width $L_{1} \approx n$ and separation distance $L_{0} \approx m$ exist. Furthermore, for each fixed $n$, these curves converge in the symmetric Hausdorff distance to the boundaries of the sets $Q_{j}(n)$ as $m \rightarrow \infty$.

The theorem is vague on how the integers $m$ and $n$ relate to the transition times $L_{0}$ and $L_{1}$. Roughly speaking, $n$ measures how many times the 2-homoclinic orbits winds around the periodic orbit, while $m$ measures how many times the 2-homoclinic orbit winds around the equilibrium during its passage near $u=0$ : recall that we assumed that $u=0$ is a bi-focus. The precise relation between $(m, n)$ and $\left(L_{0}, L_{1}\right)$ will become clear during the proof in $\S 3$.

For each fixed pair $(m, n)$, there are four symmetric 2-pulse isolas in the union $Q_{0}(n) \cup Q_{1}(n)$. As we shall see in the course of the proof, the profiles of the associated symmetric 2-pulses along these four isolas differ in the following way. For $j=1, \ldots, 4$, let $u^{(j)}(0) \in \operatorname{Fix}(\mathcal{R})$ denotes the value of the symmetric 2-pulse on the $j$ th isola at its point of symmetry, then $u^{(j)}(0)$ lies in the $j$ th quadrant of the two-dimensional space $\operatorname{Fix}(\mathcal{R})$ upon ordering the four isolas appropriately and choosing an appropriate coordinate system in $\operatorname{Fix}(\mathcal{R})$. In particular, for the Swift-Hohenberg equation, we can take $\left(U, U_{x x}\right)$ as the coordinates in $\operatorname{Fix}(\mathcal{R})$, so that $x=0$ corresponds to a positive or negative maximum or a positive or negative minimum of the profile $U(x)$ along the four isolas. An analogous statement is true for the four isolas in the set $Q_{2}(n) \cup Q_{3}(n)$. 


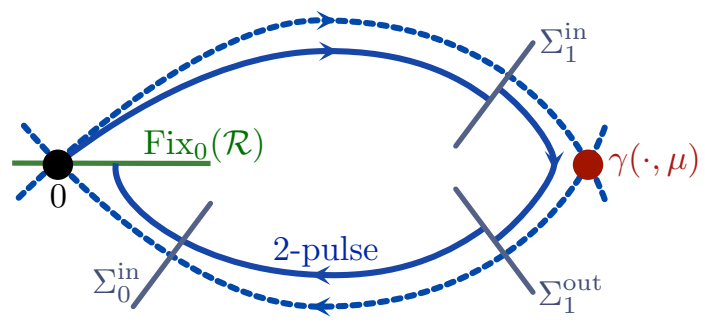

Figure 7: Illustrated are the underlying heteroclinic cycle between $u=0$ and the periodic orbit $\gamma$, the location of the various sections we shall use, the fixed-point space $\operatorname{Fix}_{0}(\mathcal{R})$ near $u=0$, and three solution pieces that we match together to construct 2-pulses.

\section{Bifurcation equations for symmetric 2-pulses}

We are interested in constructing symmetric 2-homoclinic orbits to the equilibrium $u=0$ that remain for a prescribed time $2 L_{0}$ near the equilibrium $u=0$ and for $2 L_{1}$ time units near the periodic orbit $\gamma(\cdot, \mu)$. Our strategy for finding 2-pulses is illustrated in Figure 7 and will now be outlined in more detail. Let $\operatorname{Fix}_{0}(\mathcal{R})$ be the fixed-point space of $\mathcal{R}$ restricted to some neighborhood of the equilibrium $u=0$, and denote by $\operatorname{Fix}_{1}^{\text {out }}(\mathcal{R})$ the pull-back of $\operatorname{Fix}_{0}(\mathcal{R})$ to the section $\Sigma_{1}^{\text {out }}$ under the flow. Symmetric 2-homoclinic orbits then correspond to intersections of $\operatorname{Fix}_{1}^{\text {out }}(\mathcal{R})$ with the unstable manifold $W^{u}(0, \mu)$ of the equilibrium $u=0$ tracked through a neighborhood of the periodic orbit $\gamma(\cdot, \mu)$. We will prove that $\operatorname{Fix}_{1}^{\text {out }}(\mathcal{R})$ is $C^{1}$-close to the stable manifold $W^{s}(0, \mu)$ of $u=0$. As we shall see, this property implies that the bifurcation equations for symmetric 2-homoclinic orbits can be viewed as symmetry-breaking perturbations of the bifurcation equations for 1-homoclinic orbits. An essential feature is that these perturbations do not change sign, which will imply that symmetric 2-homoclinic orbits exist along closed curves in the $(\varphi, L)$-plane as illustrated in Figure 6. On a technical level, we use a smooth, reversible, symplectic transformation into normal form to capture the dynamics near $u=0$, while we shall use the Fenichel coordinates from Lemma 2.1 to track solutions as they pass near the periodic orbit.

\subsection{Dynamics near the origin}

We first consider the local dynamics near $u=0$. Hypotheses (H1)-(H3) together with the results in [3, 19, 20] imply that there is a smooth reversible symplectic coordinate transformation from $u \in \mathbb{R}^{4}$ to new coordinates $(p, q) \in \mathbb{R}^{2} \times \mathbb{R}^{2}$ with $p=\left(p_{1}, p_{2}\right)$ and $q=\left(q_{1}, q_{2}\right)$ such that the Hamiltonian $\mathcal{H}$ in the new variables is given by

$$
\mathcal{H}(p, q, \mu)=\tilde{\mathcal{H}}(\iota, \mu)=\alpha_{0}(\mu) \iota_{1}+\beta_{0}(\mu) \iota_{2}+\mathrm{O}\left(|\iota|^{2}\right)
$$

where

$$
\iota_{1}=p_{1} q_{1}+p_{2} q_{2}, \quad \iota_{2}=p_{1} q_{2}-p_{2} q_{1}
$$

are invariants, while the reverser $\mathcal{R}$ is given by

$$
\mathcal{R}\left(p_{1}, p_{2}, q_{1}, q_{2}\right)=\left(q_{2}, q_{1}, p_{2}, p_{1}\right) .
$$

Using the above normal form of the Hamiltonian and the symplectic form $\mathcal{J}$, we see that the differential equation near $u=0$ becomes

$$
p_{x}=\left[\left(\begin{array}{cc}
-\alpha_{0}(\mu) & \beta_{0}(\mu) \\
-\beta_{0}(\mu) & -\alpha_{0}(\mu)
\end{array}\right)+\mathrm{O}(|\iota|)\right] p, \quad q_{x}=\left[\left(\begin{array}{cc}
\alpha_{0}(\mu) & \beta_{0}(\mu) \\
-\beta_{0}(\mu) & \alpha_{0}(\mu)
\end{array}\right)+\mathrm{O}(|\iota|)\right] q .
$$

In particular, the local stable manifold $W^{s}(0, \mu)$ of $u=0$ is given by $W^{s}(0, \mu)=\{(p, 0)$ : $p$ near zero $\}$. Alternatively, we can use the polar coordinates $p=r \mathrm{e}^{\mathrm{i} \phi}$ and the invariants $\iota \in \mathbb{R}^{2}$ to write the differential 


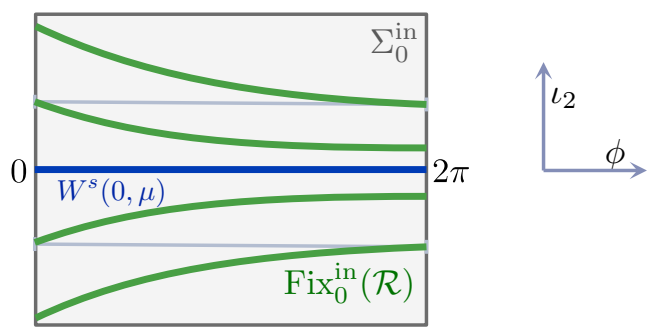

Figure 8: Shown are two of the four curves whose union is the pull-back $\operatorname{Fix}_{0}^{\text {in }}(\mathcal{R})$ of $\operatorname{Fix}_{0}(\mathcal{R})$ in the section $\Sigma_{0}^{\text {in }}$ that is parametrized by $\left(\varphi, \iota_{2} \in S^{1} \times I\right.$. Note that the section is a cylinder, so that the vertical lines $\varphi=0$ and $\varphi=2 \pi$ are identified, and that $\iota_{2}=0$ corresponds to the stable manifold $W^{s}(0, \mu)$.

equation near $(p, q)=0$ as

$$
\begin{aligned}
r_{x} & =-\tilde{\mathcal{H}}_{\iota_{1}}(\iota, \mu) r=-\left[\alpha_{0}(\mu)+\mathrm{O}(\iota)\right] r \\
\phi_{x} & =-\tilde{\mathcal{H}}_{\iota_{2}}(\iota, \mu)=-\beta_{0}(\mu)+\mathrm{O}(\iota) \\
\iota_{x} & =0 .
\end{aligned}
$$

Our first aim is to define a transverse section $\Sigma_{0}^{\text {in }}$ centered at $r=\delta$ inside $\mathcal{H}^{-1}(0)$. First, the zero level set of $\mathcal{H}$ is determined by

$$
\mathcal{H}(p, q, \mu)=\tilde{\mathcal{H}}(\iota, \mu)=\alpha_{0}(\mu) \iota_{1}+\beta_{0}(\mu) \iota_{2}+\mathrm{O}\left(|\iota|^{2}\right)=0,
$$

which can be solved uniquely for $\iota_{1}$ as a function of $\left(\iota_{2}, \mu\right)$ to get

$$
\iota_{1}=\iota_{1}^{*}\left(\iota_{2}, \mu\right)=-\frac{\beta_{0}(\mu)}{\alpha_{0}(\mu)} \iota_{2}+\mathrm{O}\left(\left|\iota_{2}\right|^{2}\right) .
$$

The desired two-dimensional section $\Sigma_{0}^{\text {in }}$ inside the zero level set of $\mathcal{H}$ is now given by

$$
\Sigma_{0}^{\text {in }}:=\left\{\left(r, \phi, \iota_{1}, \iota_{2}\right)=\left(\delta, \phi, \iota_{1}^{*}\left(\iota_{2}, \mu\right), \iota_{2}\right):\left(\phi, \iota_{2}\right) \in S^{1} \times I_{0}\right\}
$$

with $\mu \in J$, where $I_{0}$ is a sufficiently small open interval that contains zero. Next, we consider the fixed-point space $\operatorname{Fix}_{0}(\mathcal{R})$ of the reverser near the origin. In the new variables $(r, \phi, \iota)$, we have

$$
\operatorname{Fix}_{0}(\mathcal{R})=\left\{(p, q): q=\left(p_{2}, p_{1}\right), p \in \mathbb{R}^{2}\right\}=\left\{(r, \phi, \iota): \iota=\left(2 p_{1} p_{2}, p_{1}^{2}-p_{2}^{2}\right)=r^{2}(\sin 2 \phi, \cos 2 \phi)\right\} .
$$

Solving

$$
\iota_{1}=\iota_{1}^{*}\left(\iota_{2}, \mu\right), \quad \iota=r^{2}(\sin 2 \phi, \cos 2 \phi),
$$

for $\phi$ as a function of $(r, \mu)$, we obtain four solution branches given by

$$
\phi=\phi_{j}^{*}(r, \mu)=\phi_{j}^{*}(\mu)+\mathrm{O}\left(r^{2}\right), \quad j=0, \ldots, 3,
$$

where

$$
\phi_{j}^{*}(\mu):=\phi^{*}(\mu)+\frac{j \pi}{2}, \quad \phi^{*}(\mu):=-\frac{1}{2} \arctan \frac{\beta_{0}(\mu)}{\alpha_{0}(\mu)} \in\left(\frac{\pi}{4}, \frac{\pi}{2}\right)
$$

for $j=0, \ldots, 3$. In particular, we have

$$
\operatorname{Fix}_{0}(\mathcal{R}) \cap \mathcal{H}^{-1}(0)=\bigcup_{j=0, \ldots, 3}\left\{(r, \phi, \iota): \iota=r^{2}\left(\sin 2 \phi_{j}^{*}(r, \mu), \cos 2 \phi_{j}^{*}(r, \mu)\right), \phi=\phi_{j}^{*}(r, \mu), 0 \leq r \leq r_{0}\right\} .
$$

Note that the curves associated with $j=0, \ldots, 3$ lie in the $(j+1)$ th quadrant of the two-dimensional fixed-point space $\operatorname{Fix}(\mathcal{R})$. We now pull these curves back until they intersect the section $\Sigma_{0}^{\text {in }}$ that we defined above. 
Lemma 3.1 The pull-back $\operatorname{Fix}_{0}^{\text {in }}(\mathcal{R})$ of $\operatorname{Fix}_{0}(\mathcal{R}) \cap \mathcal{H}^{-1}(0)$ under the flow to the section $\Sigma_{0}^{\text {in }}$ is the union of the four curves

$$
\left(\phi, \iota_{2}\right)=\left(\tilde{\phi}_{j}^{*}, \tilde{\iota}_{j}^{*}\right)\left(L_{0}, \mu\right), \quad L_{0} \gg 1, \quad \mu \in J
$$

with

$$
\begin{aligned}
\tilde{\phi}_{j}^{*}\left(L_{0}, \mu\right) & =\phi_{j}^{*}(\mu)+\beta_{0}(\mu) L_{0}+\mathrm{O}\left(L_{0} \mathrm{e}^{-2 \alpha_{0}(\mu) L_{0}}\right) \\
\tilde{\iota}_{j}^{*}\left(L_{0}, \mu\right) & =\delta^{2} \cos \left(2 \phi_{j}^{*}(\mu)\right) \mathrm{e}^{-2 \alpha_{0}(\mu) L_{0}}\left[1+\mathrm{O}\left(\mathrm{e}^{-2 \alpha_{0}(\mu) L_{0}}\right)\right],
\end{aligned}
$$

for $j=0, \ldots, 3$, where $L_{0} \gg 1$ denotes the time needed for the associated solution to go from $\Sigma_{0}^{\mathrm{in}}$ to $\operatorname{Fix}_{0}(\mathcal{R})$.

Since

$$
W_{\mathrm{loc}}^{s}(0, \mu) \cap \Sigma_{0}^{\mathrm{in}}=\left\{(r, \phi, \iota)=(\delta, \phi, 0,0): \phi \in S^{1}\right\},
$$

we see that the set $\operatorname{Fix}_{0}^{\text {in }}(\mathcal{R})$, which is parametrized by $L_{0}$, is pointwise $\mathrm{O}\left(L_{0} \mathrm{e}^{-2 \alpha_{0}(\mu) L_{0}}\right)$-close to $W_{\text {loc }}^{s}(0, \mu)$, and we refer to Figure 8 for an illustration of the geometry.

Proof. Recall from (3.1) that $\iota(x)=\iota$ is independent of $x$. We require that $\left(r\left(L_{0}\right), \phi\left(L_{0}\right), \iota\right) \in \operatorname{Fix}_{0}\left(\mathcal{R} \cap \mathcal{H}^{-1}(0)\right.$, which gives

$$
\iota=r\left(L_{0}\right)^{2}\left(\sin 2 \phi\left(L_{0}\right), \cos 2 \phi\left(L_{0}\right)\right), \quad \phi\left(L_{0}\right)=\phi_{j}^{*}(\mu)+\mathrm{O}\left(r\left(L_{0}\right)^{2}\right) .
$$

Next, we solve (3.1) with $r(0)=\delta$ to get

$$
r(x)=\delta \mathrm{e}^{-\left[\alpha_{0}(\mu)+\mathrm{O}\left(r\left(L_{0}\right)^{2}\right)\right] x}, \quad \phi(x)=\phi(0)-\left[\beta_{0}(\mu)+\mathrm{O}\left(r\left(L_{0}\right)^{2}\right)\right] x
$$

for $0 \leq x \leq L_{0}$. Thus, we obtain

$$
r\left(L_{0}\right)=\delta \mathrm{e}^{-\alpha_{0}(\mu) L_{0}}\left[1+\mathrm{O}\left(\mathrm{e}^{-2 \alpha_{0}(\mu) L_{0}}\right)\right]
$$

and therefore

$$
\phi\left(L_{0}\right)=\phi(0)-\left[\beta_{0}(\mu)+\mathrm{O}\left(\mathrm{e}^{-2 \alpha_{0}(\mu) L_{0}}\right)\right] L_{0} .
$$

The condition (3.2) gives

$$
\phi(0)=\phi_{j}^{*}(\mu)+\beta_{0}(\mu) L_{0}+\mathrm{O}\left(L_{0} \mathrm{e}^{-2 \alpha_{0}(\mu) L_{0}}\right),
$$

and the assertions in the lemma follow now easily.

\subsection{The transition map from $\Sigma_{0}^{\text {in }}$ to $\Sigma_{1}^{\text {out }}$}

Our goal is to characterise the pull-back $\operatorname{Fix}_{1}^{\text {out }}(\mathcal{R}) \subset \Sigma_{1}^{\text {out }}$ of the set $\operatorname{Fix}_{0}^{\text {in }}(\mathcal{R})$ under the flow. Hypothesis $(\mathrm{H} 6)$ implies that the transition map

$$
\mathcal{T}: \quad \Sigma_{0}^{\text {in }} \longrightarrow \Sigma_{1}^{\text {out }}
$$

that is induced by the backward flow can be written as

$$
\left(v^{c}, v^{s}\right)=\mathcal{T}\left(\phi, \iota_{2}, \mu\right)=\left(\varphi^{*}(\phi, \mu), g\left(\varphi^{*}(\phi, \mu), \mu\right)\right)+b\left(\phi, \iota_{2}, \mu\right) \iota_{2},
$$

where $b\left(\varphi, \iota_{2}, \mu\right)$ is smooth. The map $\varphi^{*}(\cdot, \mu): S^{1} \rightarrow S^{1}$ is a diffeomorphism for each $\mu \in J$ and can therefore be written as

$$
\varphi^{*}(\phi, \mu)=\sigma[\phi+a(\phi, \mu)],
$$

where $\sigma= \pm 1$ indicates whether the diffeomorphism $\varphi^{*}(\cdot, \mu)$ preserves orientation or not and where $a(\phi, \mu)$ is $2 \pi$-periodic in $\phi$ with $a_{\phi}(\phi, \mu)>-1$ for all $(\phi, \mu)$. It will be convenient to put the transition map into the form

$$
\left(\varphi_{1}, g\left(\varphi_{1}, \mu\right)+v_{1}^{s}\right)=\mathcal{T}\left(\phi, \iota_{2}, \mu\right)
$$




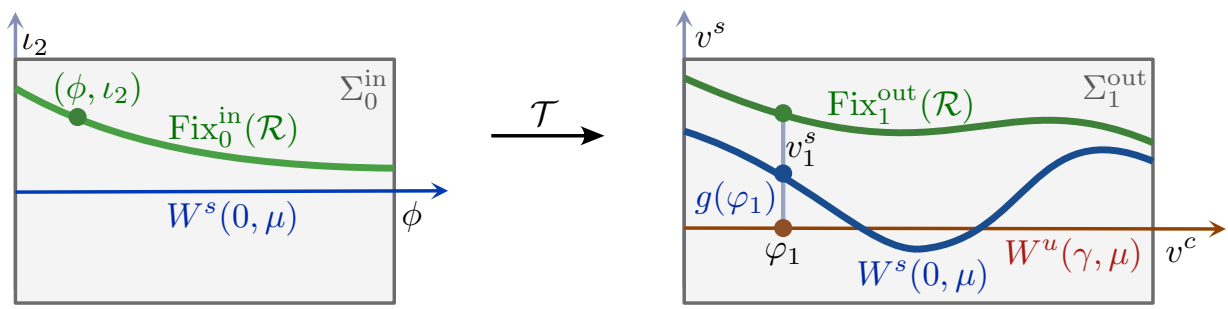

Figure 9: Illustrated is the action of $\mathcal{T}$ on $\operatorname{Fix}_{0}^{\text {in }}(\mathcal{R})$ and the interpretation of the decomposition $\mathcal{T}\left(\phi, \iota_{2}, \mu\right)=$ $\left(\varphi_{1}, g\left(\varphi_{1}, \mu\right)+v_{1}^{s}\right)$.

for appropriate values of $\left(\varphi_{1}, v_{1}^{s}\right)$; see Figure 9 . Writing $b=\left(b^{c}, b^{s}\right)$, we find that

$$
\varphi_{1}=\varphi^{*}(\phi, \mu)+b^{c}\left(\phi, \iota_{2}, \mu\right) \iota_{2}
$$

and

$$
\begin{aligned}
v_{1}^{s} & =g\left(\varphi^{*}(\phi, \mu), \mu\right)+b^{s}\left(\phi, \iota_{2}, \mu\right) \iota_{2}-g\left(\varphi_{1}, \mu\right) \\
& =g\left(\varphi^{*}(\phi, \mu), \mu\right)-g\left(\varphi^{*}(\phi, \mu)+b^{c}\left(\phi, \iota_{2}, \mu\right) \iota_{2}, \mu\right)+b^{s}\left(\phi, \iota_{2}, \mu\right) \iota_{2} \\
& =-g_{\varphi}\left(\varphi^{*}(\phi, \mu), \mu\right) b^{c}\left(\phi, \iota_{2}, \mu\right) \iota_{2}+\mathrm{O}\left(\left|\iota_{2}\right|^{2}\right)+b^{s}\left(\phi, \iota_{2}, \mu\right) \iota_{2} \\
& =\left[b^{s}(\phi, 0, \mu)-g_{\varphi}\left(\varphi^{*}(\phi, \mu), \mu\right) b^{c}(\phi, 0, \mu)+\mathrm{O}\left(\left|\iota_{2}\right|\right)\right] \iota_{2} \\
& =\left[d(\phi, \mu)+\mathrm{O}\left(\left|\iota_{2}\right|\right)\right] \iota_{2},
\end{aligned}
$$

where

$$
d(\phi, \mu):=\frac{\operatorname{det} D_{\left(\phi, \iota_{2}\right)} \mathcal{T}(\phi, 0, \mu)}{D_{\phi} \varphi^{*}(\phi, \mu)}
$$

is $2 \pi$-periodic in $\phi$ with $|d(\phi, \mu)| \geq \eta>0$ for $(\phi, \mu) \in S^{1} \times J$.

Lemma 3.1 shows that the set $\operatorname{Fix}_{0}^{\text {in }}(\mathcal{R}) \subset \Sigma_{0}^{\text {in }}$, in whose image under $\mathcal{T}$ we are interested, consists of the four curves

$$
\begin{aligned}
\phi & =\tilde{\phi}_{j}^{*}\left(L_{0}, \mu\right)=\phi_{j}^{*}(\mu)+\beta_{0}(\mu) L_{0}+\mathrm{O}\left(L_{0} \mathrm{e}^{-2 \alpha_{0}(\mu) L_{0}}\right) \\
\iota_{2} & =\tilde{\iota}_{j}^{*}\left(L_{0}, \mu\right)=\delta^{2} \cos \left(2 \phi_{j}^{*}(\mu)\right) \mathrm{e}^{-2 \alpha_{0}(\mu) L_{0}}\left[1+\mathrm{O}\left(\mathrm{e}^{-2 \alpha_{0}(\mu) L_{0}}\right)\right]
\end{aligned}
$$

with $j=0, \ldots, 3$, where $L_{0} \gg 1$ and $\mu \in J$. Substituting this parametrization of $\operatorname{Fix}_{0}^{\text {in }}(\mathcal{R})$ into the expression for $\left(\varphi_{1}, v_{1}^{s}\right)$ and using $(3.3)$, we obtain that the pull-back $\operatorname{Fix}_{1}^{\text {out }}(\mathcal{R}) \subset \Sigma_{1}^{\text {out }}$ is given by

$$
\operatorname{Fix}_{1}^{\text {out }}(\mathcal{R}): \quad\left(v^{c}, v^{u}\right)=\left(\varphi_{1}, g\left(\varphi_{1}, \mu\right)+v_{1}^{s}\right)
$$

with

$$
\begin{aligned}
\varphi_{1}=\varphi_{1}\left(L_{0}, \mu\right) & =\varphi^{*}(\phi, \mu)+b^{c}\left(\phi, \iota_{2}, \mu\right) \iota_{2} \\
& =\sigma\left[\phi_{j}^{*}(\mu)+\beta_{0}(\mu) L_{0}+a\left(\phi_{j}^{*}(\mu)+\beta_{0}(\mu) L_{0}, \mu\right)+\mathrm{O}\left(L_{0} \mathrm{e}^{-2 \alpha_{0}(\mu) L_{0}}\right)\right] \\
v_{1}^{s}=v_{1}^{s}\left(L_{0}, \mu\right) & =\left[d(\phi, \mu)+\mathrm{O}\left(\left|\iota_{2}\right|\right)\right] \iota_{2} \\
& =\underbrace{\delta^{2} \cos \left(2 \phi_{j}^{*}(\mu)\right)\left[d\left(\sigma\left(\phi_{j}^{*}(\mu)+\beta_{0}(\mu) L_{0}\right), \mu\right)+\mathrm{O}\left(L_{0} \mathrm{e}^{-2 \alpha_{0}(\mu) L_{0}}\right)\right]}_{=: d_{j}\left(L_{0}, \mu\right)} \mathrm{e}^{-2 \alpha_{0}(\mu) L_{0}},
\end{aligned}
$$

where $\left|d_{j}\left(L_{0}, \mu\right)\right| \geq \eta>0$ uniformly in $L_{0} \gg 1$ and $\mu \in J$. 


\subsection{Matching near the periodic orbit}

Using the results obtained above, the problem of finding symmetric 2-homoclinic orbits of (2.1) is now equivalent to finding solutions $v(x)$ of $(2.2)$ in $S^{1} \times I \times I$ that satisfy

$$
v\left(-L_{1}\right) \in W^{u}(0, \mu) \cap \Sigma_{1}^{\text {in }}, \quad v\left(L_{1}\right) \in \operatorname{Fix}_{1}^{\text {out }}(\mathcal{R}) \subset \Sigma_{1}^{\text {out }}
$$

for some $L_{1} \gg 1$. The next lemma gives a detailed description of solutions that stay near the periodic orbit for sufficiently large prescribed times.

Lemma $3.2([2])$ There exist positive constants $L_{*}$ and $\kappa$ so that the following is true: For each $L_{1}>L_{*}$, $\varphi \in S^{1}$ and $\mu \in J$, there is a unique solution $v(x)$, also referred to as $v(x, \varphi, \mu)$, of (2.2) that is defined for $x \in\left[-L_{1}, L_{1}\right]$ and satisfies

$$
v\left(-L_{1}\right) \in \Sigma_{1}^{\text {in }}, \quad v\left(L_{1}\right) \in \Sigma_{1}^{\text {out }}, \quad v^{c}(0)=\varphi, \quad v(x) \in S^{1} \times I \times I, \quad \forall x \in\left[-L_{1}, L_{1}\right] .
$$

Furthermore, we have

$$
\begin{aligned}
v\left(-L_{1}\right) & =\left(\varphi-L_{1}+\mathrm{O}\left(\mathrm{e}^{-\kappa L_{1}}\right), \delta, \delta \mathrm{e}^{-2 \alpha_{1}(\mu) L_{1}}\left(1+\mathrm{O}\left(\mathrm{e}^{-\kappa L_{1}}\right)\right)\right) \\
v\left(L_{1}\right) & =\left(\varphi+L_{1}+\mathrm{O}\left(\mathrm{e}^{-\kappa L_{1}}\right), \delta \mathrm{e}^{-2 \alpha_{1}(\mu) L_{1}}\left(1+\mathrm{O}\left(\mathrm{e}^{-\kappa L_{1}}\right)\right), \delta\right) \\
v(0) & =\left(\varphi, \delta \mathrm{e}^{-\alpha_{1}(\mu) L_{1}}\left(1+\mathrm{O}\left(\mathrm{e}^{-\kappa L_{1}}\right)\right), \delta \mathrm{e}^{-\alpha_{1}(\mu) L_{1}}\left(1+\mathrm{O}\left(\mathrm{e}^{-\kappa L_{1}}\right)\right)\right) .
\end{aligned}
$$

The solution $v(x)$ is smooth in $\left(L_{1}, \varphi, \mu\right)$, and the error estimates in (3.8) can be differentiated. Furthermore, we have

$$
v(x,-\varphi, \mu)=\mathcal{R} v(-x, \varphi, \mu), \quad \varphi \in S^{1}, \quad|x| \leq L_{1}, \quad \mu \in J .
$$

In particular, the solution $v(x, \varphi, \mu)$ is $\mathcal{R}$-reversible with $v(0) \in \operatorname{Fix}(\mathcal{R})$ if and only if $\varphi \in \Theta$.

Lemma 3.2 shows that $v(x)=v(x, \varphi, \mu)$ for an appropriate value of $\varphi \in S^{1}$, while (3.9) implies that $v\left(-L_{1}, \varphi, \mu\right) \in$ $W^{u}(0, \mu) \cap \Sigma_{1}^{\text {in }}$ if and only if $v\left(L_{1},-\varphi, \mu\right) \in W^{s}(0, \mu) \cap \Sigma_{1}^{\text {out }}$. Thus, using also Hypothesis (H6) and (3.4)-(3.6), we see that (3.7) is equivalent to

$$
\begin{aligned}
v^{s}\left(L_{1},-\varphi, \mu\right) & =g\left(v^{c}\left(L_{1},-\varphi, \mu\right), \mu\right) \\
v^{s}\left(L_{1}, \varphi, \mu\right) & =g\left(\varphi_{1}\left(L_{0}, \mu\right), \mu\right)+v_{1}^{s}\left(L_{0}, \mu\right) \\
v^{c}\left(L_{1}, \varphi, \mu\right) & =\varphi_{1}\left(L_{0}, \mu\right) .
\end{aligned}
$$

Instead of this system, we will use the equivalent, but slightly more convenient, formulation

$$
\begin{aligned}
v^{s}\left(L_{1},-\varphi, \mu\right) & =g\left(v^{c}\left(L_{1},-\varphi, \mu\right), \mu\right) \\
v^{s}\left(L_{1}, \varphi, \mu\right) & =g\left(v^{c}\left(L_{1}, \varphi, \mu\right), \mu\right)+v_{1}^{s}\left(L_{0}, \mu\right) \\
v^{c}\left(L_{1}, \varphi, \mu\right) & =\varphi_{1}\left(L_{0}, \mu\right) .
\end{aligned}
$$

We remark that $\left(\varphi_{1}, v_{1}^{s}\right)$ depends on $j=0, \ldots, 3$, but we will fix $j$ from now on and omit the dependence on $j$.

First, we solve (3.10). Using the expansions for $\left(v^{c}, v^{s}\right)$ from (3.8), the fact (2.4) that $g(\varphi, \mu)=0$ if and only if $\mu=z(\varphi)$, and the property $\left|g_{\mu}(\varphi, \mu)\right| \geq \eta>0$ from Hypothesis (H6), we obtain via the implicit function theorem that there is a function $e\left(L_{1}, \varphi\right)$ such that (3.10) holds if and only if

$$
\mu=\mu_{*}\left(L_{1}, \varphi\right):=z\left(L_{1}-\varphi\right)+e\left(L_{1},-\varphi\right), \quad e\left(L_{1}, \varphi\right)=\mathrm{O}\left(\mathrm{e}^{-\kappa L_{1}}\right)
$$

uniformly in $L_{1} \gg 1$ and $\varphi \in S^{1}$. 

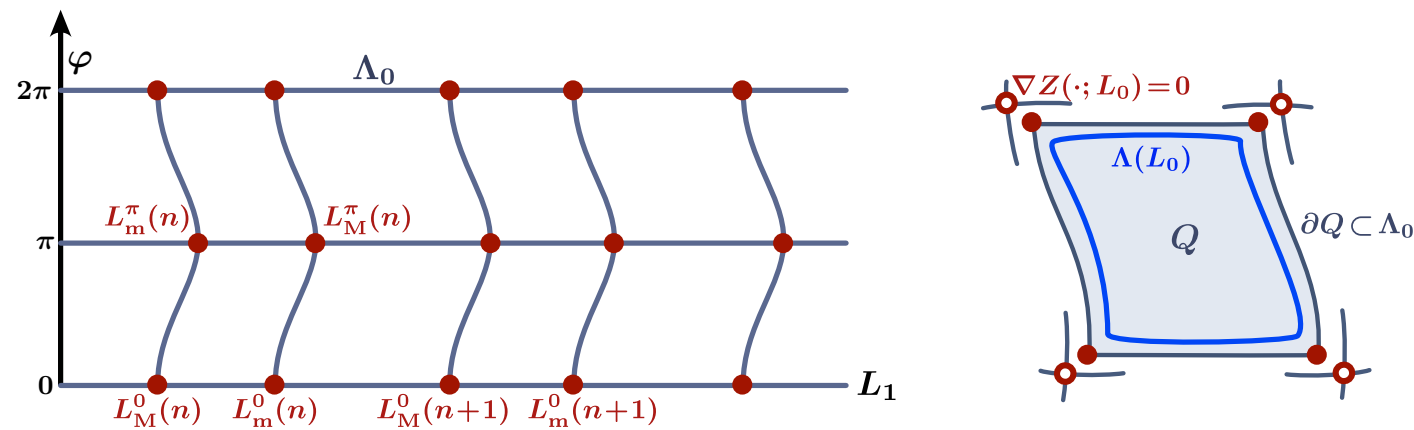

Figure 10: The left panel illustrates the zero level set $\Lambda_{0}$ of the function $Z_{0}\left(L_{1}, \varphi\right)$ in the cylinder $\left(L_{*}, \infty\right) \times S^{1}$. The set $\Lambda_{0}$ contains the horizontal lines $\varphi=0$ and $\varphi=\pi$ as well as infinitely many connecting rung curves that begin and end at the critical points $L_{1}=L_{\mathrm{m}, \mathrm{M}}^{0, \pi}(n)$ along the horizontal lines. The right panel shows one of the rectangles $Q$ enclosed by $\Lambda_{0}$ together with the critical points and the zero level set $\Lambda\left(L_{0}\right)$ of $Z\left(\cdot ; L_{0}\right)$ for fixed $L_{0}$.

Next, consider (3.11) and note that the unique solution of (3.11) with $v_{1}^{s}=0$ is given by $\mu=z\left(L_{1}+\varphi\right)+e\left(L_{1}, \varphi\right)$. Thus, we set

$$
\mu=z\left(L_{1}+\varphi\right)+e\left(L_{1}, \varphi\right)+\mu_{2}=: \mu_{1}\left(L_{1}, \varphi\right)+\mu_{2}
$$

and consider (3.11) in the form

$$
g\left(v^{c}\left(L_{1}, \varphi, \mu\right), \mu\right)-v^{s}\left(L_{1}, \varphi, \mu\right)+v_{1}^{s}\left(L_{0}, \mu\right)=0,
$$

which then becomes

$$
\begin{aligned}
& g\left(v^{c}\left(L_{1}, \varphi, \mu_{1}\left(L_{1}, \varphi\right)+\mu_{2}\right), \mu_{1}\left(L_{1}, \varphi\right)+\mu_{2}\right)-v^{s}\left(L_{1}, \varphi, \mu_{1}\left(L_{1}, \varphi\right)+\mu_{2}\right) \\
& \quad+v_{1}^{s}\left(L_{0}, \mu_{1}\left(L_{1}, \varphi\right)+\mu_{2}\right)-v_{1}^{s}\left(L_{0}, \mu_{1}\left(L_{1}, \varphi\right)\right)=-v_{1}^{s}\left(L_{0}, \mu_{1}\left(L_{1}, \varphi\right)\right) .
\end{aligned}
$$

The preceding discussion shows that the left-hand side vanishes when $\mu_{2}=0$, and (3.15) can therefore be written as

$$
\tilde{g}\left(L_{0}, L_{1}, \varphi, \mu_{2}\right) \mu_{2}=-v_{1}^{s}\left(L_{0}, \mu_{1}\left(L_{1}, \varphi\right)\right),
$$

where

$$
\tilde{g}\left(L_{0}, L_{1}, \varphi, \mu_{2}\right)=g_{\mu}\left(L_{1}+\varphi, z\left(L_{1}+\varphi\right)\right)+\mathrm{O}\left(\mathrm{e}^{-\kappa L_{0}}+\mathrm{e}^{-\kappa L_{1}}+\left|\mu_{2}\right|\right)
$$

for some positive $\kappa$ uniformly in $L_{0}, L_{1} \gg 1, \varphi \in S^{1}$, and $\left|\mu_{2}\right|$ small. Equation (3.6) shows that

$$
v_{1}^{s}\left(L_{0}, \mu_{1}\left(L_{1}, \varphi\right)\right)=d_{j}\left(L_{0}, \mu_{1}\left(L_{1}, \varphi\right)\right) \mathrm{e}^{-2 \alpha_{0}\left(\mu_{1}\left(L_{1}, \varphi\right)\right) L_{0}},
$$

and we conclude from (3.17) and Hypothesis (H6) that (3.16) has a unique solution $\mu_{2}=\mu_{2}\left(L_{0}, L_{1}, \varphi\right)$ and that this solution has the expansion

$$
\mu_{2}\left(L_{0}, L_{1}, \varphi\right)=-\underbrace{\left[\frac{d_{j}\left(L_{0}, z\left(L_{1}+\varphi\right)\right)}{g_{\mu}\left(L_{1}+\varphi, z\left(L_{1}+\varphi\right)\right)}+\mathrm{O}\left(\mathrm{e}^{-\kappa L_{0}}+\mathrm{e}^{-\kappa L_{1}}\right)\right]}_{=: \tilde{d}_{j}\left(L_{0}, L_{1}, \varphi\right)} \mathrm{e}^{-2 \alpha_{0}\left(\mu_{1}\left(L_{1}, \varphi\right)\right) L_{0}} .
$$

Note that $\eta_{1} \geq\left|\tilde{d}_{j}\left(L_{0}, L_{1}, \varphi\right)\right| \geq \eta_{0}>0$ and that the derivatives of $\tilde{d}_{j}$ with respect to any of its arguments are bounded by $\eta_{1}$ uniformly in $L_{0}, L_{1} \gg 1$ and $\varphi \in S^{1}$.

The solution $\mu=\mu_{*}\left(L_{1}, \varphi\right)$ of (3.10) from (3.13) and the solution $\mu=\mu_{1}\left(L_{1}, \varphi\right)+\mu_{2}\left(L_{0}, L_{1}, \varphi\right)$ of $(3.11)$ from (3.14) and (3.18) need to coincide. Inspecting their expansions, we see that these solutions coincide if and only if

$$
\begin{aligned}
Z\left(L_{1}, \varphi ; L_{0}\right) & :=\mu_{1}\left(L_{1}, \varphi\right)+\mu_{2}\left(L_{0}, L_{1}, \varphi\right)-\mu_{*}\left(L_{1}, \varphi\right) \\
& =\underbrace{z\left(L_{1}+\varphi\right)+e\left(L_{1}, \varphi\right)-z\left(L_{1}-\varphi\right)-e\left(L_{1},-\varphi\right)}_{=: Z_{0}\left(L_{1}, \varphi\right)}+\mu_{2}\left(L_{0}, L_{1}, \varphi\right) \\
& =0 .
\end{aligned}
$$


Note that $Z_{0}\left(L_{1},-\varphi\right)=-Z_{0}\left(L_{1}, \varphi\right)$ for all $\left(L_{1}, \varphi\right)$, which implies that $Z_{0}\left(L_{1}, 0\right)=Z_{0}\left(L_{1}, \pi\right)=0$ for all $L_{1}$. It was shown in [2] under hypotheses that are weaker than ours that $\Lambda_{0}:=Z_{0}^{-1}(0)$ looks as shown in Figure 10. Furthermore, it was shown there that the critical points of $Z_{0}$ in $\lambda_{0}$ are given by $\left(L_{1}, \varphi\right)$ with $\varphi \in \Theta:=\{0, \pi\}$ and $L_{1}=\ell_{i}-\varphi+2 \pi n+\mathrm{O}\left(\mathrm{e}^{-\kappa n}\right)$ for $i=\mathrm{m}, \mathrm{M}$ and integers $n \gg 1$, where we recall that $\ell_{\mathrm{m}}$ and $\ell_{\mathrm{M}}$ denote, respectively, the minimum and maximum of the function $z$. Finally, it follows from Hypothesis (H5) that the critical points of $Z_{0}$ in $\Lambda_{0}$ are nondegenerate since their Hessian

$$
D^{2} Z_{0}\left(L_{1}, \varphi\right)=\left(\begin{array}{cc}
0 & 2 z^{\prime \prime}\left(\ell_{i}\right)+\mathrm{O}\left(\mathrm{e}^{-\kappa n}\right) \\
2 z^{\prime \prime}\left(\ell_{i}\right)+\mathrm{O}\left(\mathrm{e}^{-\kappa n}\right) & 0
\end{array}\right), \quad i=\mathrm{m}, \mathrm{M}
$$

is nondegenerate.

Our goal is to investigate the nature of the zero level set

$$
\Lambda\left(L_{0}\right):=\left\{\left(L_{1}, \varphi\right): Z\left(L_{1}, \varphi ; L_{0}\right)=0\right\}
$$

of $Z\left(L_{1}, \varphi ; L_{0}\right)$ for each fixed value of $L_{0} \gg 1$. Note that $\mu_{2}$ is exponentially small in $L_{0}$ so that $Z_{0}$ and $Z\left(\cdot ; L_{0}\right)$ are close for each $L_{0} \gg 1$, and we therefore expect that $\Lambda\left(L_{0}\right)$ is close to $\Lambda_{0}$. Moreover, using (3.18), we see that, for $\left(L_{1}, \varphi\right) \in \Lambda_{0}$ and $L_{0} \gg 1$, we have

$$
Z\left(L_{1}, \varphi ; L_{0}\right)=Z_{0}\left(L_{1}, \varphi\right)+\mu_{2}\left(L_{0}, L_{1}, \varphi\right)=\mu_{2}\left(L_{0}, L_{1}, \varphi\right)=\underbrace{\tilde{d}_{j}\left(L_{0}, L_{1}, \varphi\right)}_{|\ldots| \geq \eta_{0}>0} \mathrm{e}^{-2 \alpha_{0}\left(\mu_{1}\left(L_{1}, \varphi\right)\right) L_{0}} \neq 0 \quad \forall\left(L_{1}, \varphi\right) \in \Lambda_{0},
$$

so that $\Lambda_{0} \cap \Lambda\left(L_{0}\right)=\emptyset$ for all $L_{0} \gg 1$. First, we consider the critical points of $Z$ for fixed $L_{0}$, which must lie near the critical points of $Z_{0}$. Thus, pick one of the critical points $\left(L_{1}^{0}, \varphi^{0}\right)$ of $Z_{0}$ in $\Lambda_{0}$ that we discussed above and write $\left(L_{1}, \varphi\right)=\left(L_{1}^{0}, \varphi^{0}\right)+(\tilde{L}, \tilde{\varphi})$. Expanding $Z\left(L_{1}, \varphi ; L_{0}\right)$ in $(\tilde{L}, \tilde{\varphi})$ for fixed $L_{0}$ and recalling $(3.18)$ and (3.20), we see that

$$
\begin{aligned}
D_{\left(L_{1}, \varphi\right)} Z\left(L_{1}, \varphi ; L_{0}\right) & =D Z_{0}\left(L_{1}^{0}+\tilde{L}, \varphi^{0}+\tilde{\varphi}\right)+D_{\left(L_{1}, \varphi\right)} \mu_{2}\left(L_{0}, L_{1}^{0}+\tilde{L}, \varphi^{0}+\tilde{\varphi}\right) \\
& =D^{2} Z_{0}\left(L_{1}^{0}, \varphi^{0}\right)(\tilde{L}, \tilde{\varphi})+\mathrm{O}\left(\left(\mathrm{e}^{-\kappa L_{0}}+|\tilde{L}|+|\tilde{\varphi}|\right)(|\tilde{L}|+|\tilde{\varphi}|)\right)+D_{\left(L_{1}, \varphi\right)} \mu_{2}\left(L_{0}, L_{1}^{0}, \varphi^{0}\right) \\
& =0
\end{aligned}
$$

can be solved uniquely for $(\tilde{L}, \tilde{\varphi})$ for each $L_{0} \gg 1$ and that this solution satisfies

$$
(\tilde{L}, \tilde{\varphi})=\mathrm{O}\left(\mathrm{e}^{-2 \alpha_{0}\left(\mu_{1}\left(L_{1}^{0}, \varphi^{0}\right)\right) L_{0}}\right)
$$

Furthermore, the value of $Z\left(L_{1}, \varphi ; L_{0}\right)$ at these $L_{0}$-dependent critical points is given by

$$
\begin{aligned}
Z\left(L_{1}^{0}+\tilde{L}, \varphi^{0}+\tilde{\varphi} ; L_{0}\right) & =\mathrm{O}\left((|\tilde{L}|+|\tilde{\varphi}|)^{2}\right)+\mu_{2}\left(L_{0}, L_{1}^{0}+\tilde{L}, \varphi^{0}+\tilde{\varphi}\right) \\
& =\mu_{2}\left(L_{0}, L_{1}^{0}, \varphi^{0}\right)+\mathrm{O}\left(\left(\mathrm{e}^{-\kappa L_{0}}+|\tilde{L}|+|\tilde{\varphi}|\right)(|\tilde{L}|+|\tilde{\varphi}|)\right) \\
& =\tilde{d}_{j}\left(L_{0}, L_{1}^{0}, \varphi^{0}\right) \mathrm{e}^{-2 \alpha_{0}\left(\mu_{1}\left(L_{1}^{0}, \varphi^{0}\right)\right) L_{0}}+\mathrm{O}\left(\mathrm{e}^{-\kappa L_{0}} \mathrm{e}^{-2 \alpha_{0}\left(\mu_{1}\left(L_{1}^{0}, \varphi^{0}\right)\right) L_{0}}\right) \\
& =\underbrace{\left[\tilde{d}_{j}\left(L_{0}, L_{1}^{0}, \varphi^{0}\right)+\mathrm{O}\left(\mathrm{e}^{-\kappa L_{0}}\right)\right]}_{|\ldots| \geq \eta>0} \mathrm{e}^{-2 \alpha_{0}\left(\mu_{1}\left(L_{1}^{0}, \varphi^{0}\right)\right) L_{0}}
\end{aligned}
$$

uniformly in $L_{0} \gg 1$. In particular, the set $\Lambda\left(L_{0}\right)$ does not contain any critical points of $Z\left(\cdot ; L_{0}\right)$ and is therefore locally a smooth curve that we can parametrize by its arclength $s$.

Figure 10 summarizes what we have shown so far. For fixed index $j$, the quantity $\mu_{2}$ has a definite sign, and since $\Lambda_{0} \cap \Lambda\left(L_{0}\right)=\emptyset$, the associated set $\Lambda\left(L_{0}\right)$ is the disjoint union of sets that each lie strictly inside one of the bounded rectangles whose boundaries form the set $\Lambda_{0}$. Pick one of these rectangles inside which $Z\left(\cdot ; L_{0}\right)$ has the same sign as $\mu_{2}$ and denote it by $Q$. The boundary $\partial Q$ of $Q$ consists of four critical points of $Z_{0}$ and 
four curve segments with finite arclength. Outside each fixed small neighborhood of the critical points, we can solve $Z\left(L_{1}, \varphi ; L_{0}\right)=0$ by the implicit function theorem for $\left(L_{1}, \varphi\right)$ as functions of $L_{0} \gg 1$ and the arclength parameter $s$ along the curve segments in $\partial Q$. Furthermore, these functions satisfy

$$
D_{L_{0}}\left(L_{1}, \varphi\right)\left(s, L_{0}\right)=\mathrm{O}\left(\mathrm{e}^{-\kappa L_{0}}\right)
$$

uniformly in $s$. Next, we discuss the set $\Lambda\left(L_{0}\right)$ near each of the four critical points. Pick one of these four and recall that we have shown above that $Z\left(\cdot ; L_{0}\right)$ has a unique critical point nearby. Furthermore, this $L_{0}$-dependent critical point of $Z\left(\cdot ; L_{0}\right)$ of saddle type and nondegenerate. Thus, we can apply the Morse lemma to show that, after a smooth coordinate transformation of $\left(L_{1}, \varphi\right)$ that depends also smoothly on $L_{0}$, the function $Z\left(\cdot ; L_{0}\right)$ near the critical point is given by

$$
Z\left(x, y ; L_{0}\right)=x^{2}-y^{2}-\tilde{d}_{j}\left(x, y, L_{0}\right) \mathrm{e}^{-2 \alpha_{0}(x, y) L_{0}},
$$

where $\left|\alpha_{0}\right| \geq \kappa>0,\left|\tilde{d}_{j}\right| \geq \eta>0$, and their derivatives with respect to $\left(x, y, L_{0}\right)$ are bounded. Here, $(x, y)=0$ corresponds to the critical point $\left(L_{1}, \varphi\right)=\left(L_{1}^{0}, \varphi^{0}\right)+(\tilde{L}, \tilde{\varphi})$ constructed above. It is now straightforward to solve $Z\left(x, y ; L_{0}\right)=0$ for $(x, y)$ as functions of $L_{0}$ and the arclength parameter $s$, and we again find that the associated functions $\left(L_{1}, \varphi\right)\left(s, L_{0}\right)$ satisfy the estimate (3.21). Thus, using uniqueness of solutions, the different curves we constructed near the curve segments and the critical points fit together to give a closed curve that lies inside $Q$ and is close to its boundary $\partial Q$. Furthermore, this solution curve satisfies (3.21).

In summary, we have now solved the equations (3.10)-(3.11), and it remains to solve (3.12) given by

$$
v^{c}\left(L_{1}, \varphi, \mu\right)=\varphi_{1}\left(L_{0}, \mu\right)
$$

Substituting $v^{c}$ from Lemma 3.2 and $\varphi_{1}$ from (3.5), we obtain the equation

$$
L_{1}+\varphi+\mathrm{O}\left(\mathrm{e}^{-\kappa L_{1}}\right)=\sigma\left[\phi_{j}^{*}(\mu)+\beta_{0}(\mu) L_{0}+a\left(\phi_{j}^{*}(\mu)+\beta_{0}(\mu) L_{0}, \mu\right)+\mathrm{O}\left(\mathrm{e}^{-\kappa L_{0}}\right)\right],
$$

where $\sigma= \pm 1, \mu=z\left(L_{1}-\varphi\right)+\mathrm{O}\left(\mathrm{e}^{-\kappa L_{1}}\right)$, and $\left(L_{1}, \varphi\right)=\left(L_{1}, \varphi\right)\left(s, L_{0}\right)$ are the functions constructed above. Recall that $\left(L_{1}, \varphi\right)\left(s, L_{0}\right)$ stays inside the fixed rectangle $Q$ for all values of $\left(s, L_{0}\right)$. Thus, using $(3.21)$ and the inequality $a_{\phi}(\phi, \mu)>-1$ that holds for all $(\phi, \mu)$, we can solve (3.22) uniquely for $L_{0}=L_{0}(s)$ for each fixed $s$. Since $\left(L_{1}, \varphi\right)\left(s, L_{0}\right)$ is a closed curve for each fixed $L_{0}$, the resulting solution $\left(L_{0}, L_{1}, \varphi\right)(s)$ gives closed curve as claimed.

Note that the sign of $\sigma$ has implications for how $L_{0}$ adjusts with $\left(L_{1}, \varphi\right)$ along each 2-pulse isola. Assume, for instance, that $\sigma=-1$. Upon varying $s$, the curve $\left(L_{1}, \varphi\right)(s)$ will pass near the boundary of the rectangle $Q$ shown in Figure 10. Pick the segment along which $\varphi(s)$ is approximately constant, while $L_{1}(s)$ increases, then (3.22) shows that $L_{0}(s)$ will actually decrease. Thus, for $\sigma=-1$, if one of $L_{0}$ or $L_{1}$ increases, then the other variable will decrease, and vice versa. In contrast, if $\sigma=1$, then both $L_{1}(s)$ and $L_{0}(s)$ will increase and decrease together. We remark that we have $\sigma=-1$ near the Turing bifurcations at which the periodic orbits $\gamma(\cdot, \mu)$ emerge from the origin. The computations for the Swift-Hohenberg equation (1.1) that we present in the next section indicate that $\sigma=-1$ for this equation even for parameter values further away from the Turing bifurcation.

\section{Discussion}

Summary. Motivated by numerical computations for the Swift-Hohenberg equation, we have analysed the existence of symmetric 2-pulse solutions in homoclinic snaking scenarios. Two-pulse solutions are characterised by two quantities, namely the transition time $2 L_{0}$ near the equilibrium, which measures the separation distance between the two single pulses that form the 2-pulse, and the transition time $2 L_{1}$ near the underlying periodic 

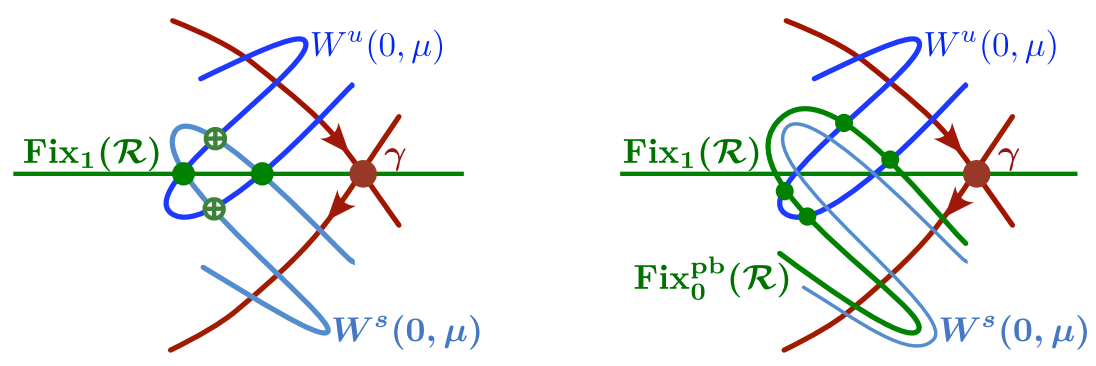

Figure 11: The two panels illustrate the dynamics of the Poincare map associated with a fixed two-dimensional transverse section placed at $\gamma(0, \mu)$ on the periodic orbit. The left panel indicates how symmetric (bullets) and non-symmetric (circled crosses) 1-homoclinic orbits to $u=0$ arise as intersections of $W^{s}(0, \mu)$ and $W^{u}(0, \mu)$ : note that symmetric 1-pulses lie in $\operatorname{Fix}_{1}(\mathcal{R})$. The right panel illustrates the location of symmetric 2-homoclinic orbits based on the observation that the pull-back $\operatorname{Fix}_{0}^{\mathrm{pb}}(\mathcal{R})$ of the fixed-point space of the reverser is close to $W^{s}(0, \mu)$.

orbit, which corresponds to the width of the single pulses. We showed that the separation distance and the width of 2-pulses can be parametrized by natural numbers $m$ and $n$ that correspond respectively to the number of oscillations near the equilibrium and to the number of windings that the 2-pulse makes along the periodic orbit. We proved that symmetric 2-pulses exist along a two-parameter family of figure-eight shaped isolas that are parametrized by the integers $(m, n)$. Varying $m$ for fixed $n$ results in a nested family of isolas, while varying $n$ for fixed $m$ gives a stacked family of isolas. Our proof was based on tracking the stable and unstable manifolds of the equilibrium along the underlying heteroclinic cycle whose existence we assumed. To track these manifolds, we used a smooth reversible and symplectic transformation into normal form near the equilibrium and Fenichel coordinates near the periodic orbit. Reversibility then implies that the bifurcation equations for symmetric 2-homoclinic orbits can be viewed as symmetry-breaking perturbations of the bifurcation equations for symmetric 1-homoclinic orbits: the effect of the symmetry-breaking terms is that the snakes-and-ladder structure of 1-pulses breaks up into figure-eight isolas of 2-pulses. This phenomenon is similar to the effect that forced symmetry-breaking has on the double helix structure of 1-pulses, which was recently explored numerically in $[5]$.

Connection with Poincare maps. A geometric way of viewing symmetric 2-homoclinic orbits consists of inspecting the dynamics of the Poincare map to a fixed two-dimensional transverse section that is placed at the underlying periodic orbit inside the zero level set of the Hamiltonian. This is illustrated in Figure 11, where the location of symmetric and non-symmetric 1-homoclinic orbits is shown in the left panel, while the location of symmetric 2-homoclinic orbits is indicated in the right panel. Upon changing the parameter $\mu$, the position of the invariant manifolds will change, and a careful analysis of the geometry in the cross section yields another explanation of homoclinic snaking; see $[2,25]$. In particular, the fact that $W^{s}(0, \mu)$ and $W^{u}(0, \mu)$ are $\mathcal{R}$-images of each other shows that the non-symmetric orbits emerge in pitchfork bifurcations very close to fold bifurcations of symmetric orbits. Symmetric 2-homoclinic orbits can be studied similarly upon making use of the fact that the pull-back $\operatorname{Fix}_{0}^{\mathrm{pb}}(\mathcal{R})$ of the fixed-point space of the reverser in the transverse section is close to the stable manifold $W^{s}(0, \mu)$ as indicated in the right panel in Figure 11. Since the pull-back closely follows $W^{s}(0, \mu)$, but is not exactly an $\mathcal{R}$-image of $W^{u}(0, \mu)$, symmetric 2-homoclinic solutions will emerge in fold and not in pitchfork bifurcations, when the curves are moved through each other as the parameter changes.

Reversibility and Hamiltonian structure. The key structural assumptions on the underlying vector field that we utilised in our analysis are its reversibility and the presence of a Hamiltonian. We now discuss briefly what our expectations are for systems that have only one or none of these structures in place.

First, for generic systems that are neither conservative nor reversible, homoclinic snaking will likely be a 


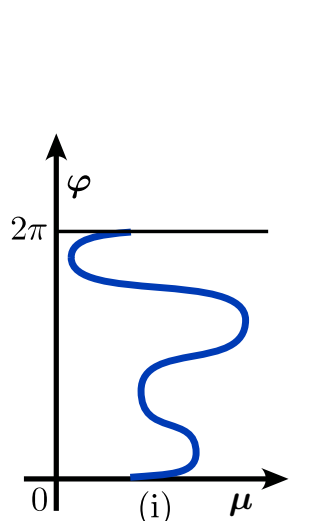

(i)
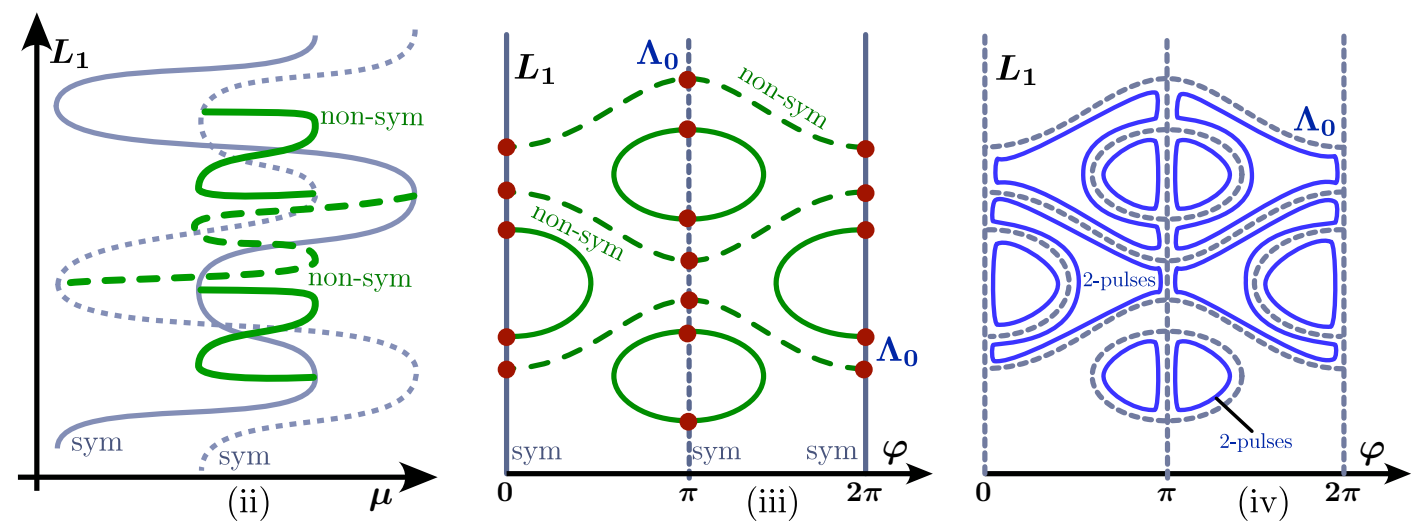

Figure 12: Panels (i) and (ii) show a function $\mu=z(\varphi)$ with more than four turning points and the resulting snaking diagram of symmetric and non-symmetric 1-pulses from [2]. Panel (iii) illustrates the corresponding zero level set $\Lambda_{0}=Z_{0}^{-1}(0)$ of the function $Z_{0}\left(L_{1}, \varphi\right)$ whose image under the map (4.1) gives panel (ii). We believe that symmetric 2-pulses exist along curves that, roughly speaking, are close to the level sets $Z_{0}^{-1}( \pm \epsilon)$ of $Z_{0}$, where $0<\epsilon \ll 1$ depends on $L_{0}$ as in $\S 3.3$. These are indicated in panel (iv).

codimension-one phenomenon. Indeed, without either of these two structures, we could not assume the generic existence of a heteroclinic cycle that consists of an orbit that connects $u=0$ at $x=-\infty$ to the roll pattern at $x=\infty$ and another orbit that connects the roll pattern at $x=-\infty$ with $u=0$ at $x=\infty$. Codimension-one snaking is discussed further in $[7,17]$ to which we refer for details.

For systems that are Hamiltonian but not reversible, heteroclinic cycles of the type discussed above exist robustly. However, the underlying fronts and backs between $u=0$ and the roll pattern may appear and disappear independently of each other. We do not know what the resulting homoclinic snaking diagrams would look like but believe that our methods should be applicable to analyse this scenario.

We do not believe that the Hamiltonian structure is essential. It was shown in $[2, \S 6.4]$ that asymmetric rung states exist even without a Hamiltonian structure; however, these structures will no longer be stationary but will instead move with nonzero speed $c$ of order $\mathrm{e}^{-\eta L_{1}}$ for some $\eta>0$ as solutions to the underlying PDE. Combining the ideas in $[2, \S 6.4]$ with the analysis presented here, it should be possible to show that stationary symmetric 2-pulses exist along figure-of-eight isolas in reversible ODEs without a Hamiltonian structure. Note that symmetric 2-pulses cannot move.

Hypotheses (H5)-(H6). We now comment on the role of Hypotheses (H5)-(H6). Snaking was established in [2] under far weaker assumptions on the geometry of the stable and unstable manifolds near the periodic orbit than those encoded in (H5)-(H6). In particular, [2, Hypotheses 6-7] covers the situation where the function $\mu=z(\varphi)$ has several nondegenerate maxima and minima. Furthermore, the modifications outlined in $[2, \S 6.1]$ allow for isolas of heteroclinic orbits and therefore apply to the case where the stable manifold $W^{s}(0, \mu)$ is not a graph over the unstable manifold $W^{u}(\gamma, \mu)$ of the periodic orbit.

We believe that our results for symmetric 2-pulses should be valid also in this more general setting without major complications in the proof. Indeed, our proof relies only on the analysis of the zero level set $\Lambda_{0}$ of the function $Z_{0}\left(L_{1}, \varphi\right)$ in the $\left(\varphi, L_{1}\right)$-plane plotted in Figures 6 and 10 and then utilises the map

$$
\left(L_{1}, \varphi\right) \mapsto\left(\mu_{*}\left(L_{1}, \varphi\right), L_{1}\right)
$$

from (3.13) to generate the complete bifurcation diagram. In particular, symmetric 2-pulses exist along the zero level set of the function $Z\left(L_{1}, \varphi\right)$ which is approximately of the form $Z\left(L_{1}, \varphi\right)=Z_{0}\left(L_{1}, \varphi\right) \pm \eta \mathrm{e}^{-2 \alpha_{0} L_{0}}$ for fixed $L_{0} \gg 1$ and $\eta>0$. Hence, the zero level set of $Z$ along which 2-pulses exist can be thought of as the level sets 

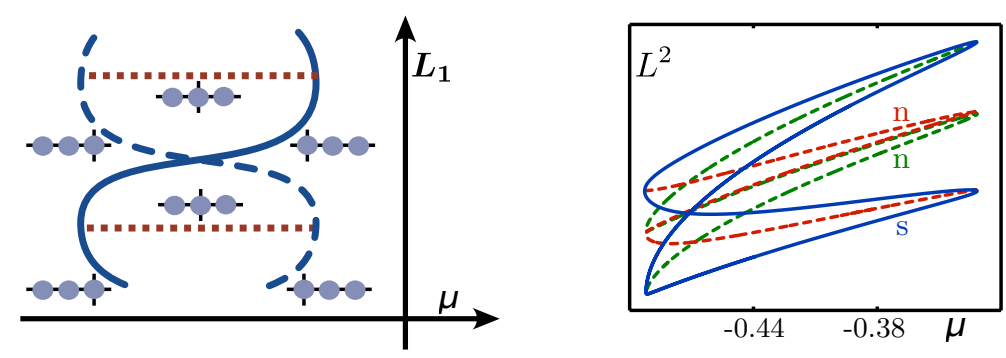

Figure 13: The left panel contains a sketch of the anticipated PDE spectra of symmetric and non-symmetric 1-pulses along part of the double helix. The right panel contains the results of numerical computations of the Swift-Hohenberg equation (1.1): shown are an isola of symmetric 2-pulses (labelled (s)) and two z-shaped branches (labelled $(n)$ ) of non-symmetric 2-pulses that emerge at pitchfork bifurcations from the symmetric 2-pulse isola.
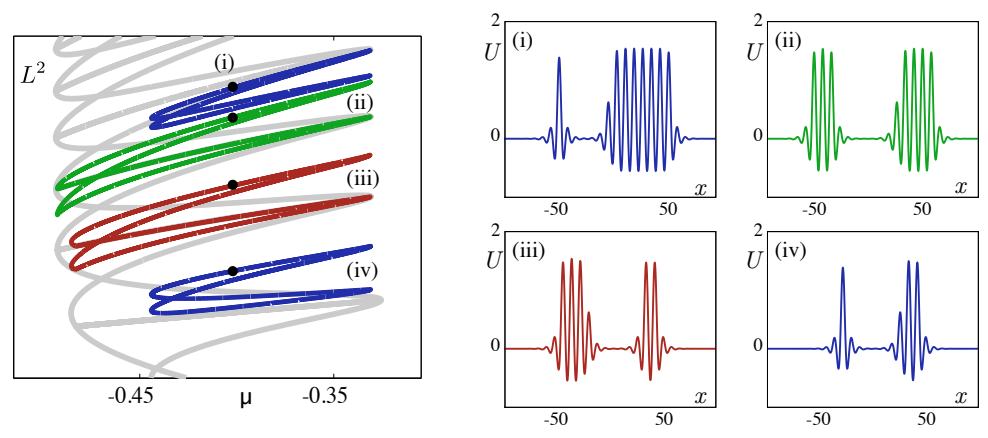

Figure 14: The left panel contains four isolas of non-symmetric 2-homoclinic orbits of (1.1) together with the 1-pulse double-helix structure plotted in light gray. Representative profiles of solutions along each of the isolas are plotted in the right four panels.

of $Z_{0}$ for values slightly higher and lower than zero; see Figure 10 for an illustration. These arguments should remain valid for more general functions $z(\varphi)$. In Figure 12, we illustrate the relevant geometry for a function $z(\varphi)$ with four turning points, which occurs in the planar Swift-Hohenberg equation [1]. We expect that symmetric 2-pulses exist along the zero level set of $Z$ which resembles the level sets $Z_{0}^{-1}( \pm \epsilon)$ of $Z_{0}$ for $\epsilon \ll 1$. We refer to Figure 12(iv) for an illustration of the anticipated bifurcation diagram of symmetric 2-pulses in the $\left(\varphi, L_{1}\right)$-plane.

Stability. Next, we comment on the anticipated stability properties of symmetric 2-pulses for the SwiftHohenberg equation. Numerical computations [4] and formal results [2] indicate that the spectrum of 1-pulses is as shown in Figure 13. The two eigenvalues that move back and forth as each snaking curve of symmetric 1-pulses is traversed cause folds and the pitchfork bifurcations to non-symmetric 1-pulses. The results in [22, 23] imply that each 1-pulse eigenvalue creates two eigenvalues of the symmetric 2-pulse that we constructed in this paper, provided $m \gg 1$ is sufficiently large; recall that $m$ parametrizes the admissible separation distances $L_{0}$ within the 2-pulse. In particular, the translation eigenvalue at zero of the 1-pulse creates the translation eigenvalue of the symmetric 2-pulse and a second eigenvalue that is stable for even indices $m$ and unstable for odd $m$ (or vice versa); see $[22,23]$. These results together with the form of the isolas of symmetric 2-pulses described here show that symmetric 2-pulses undergo pitchfork bifurcations near each of the folds along the isola on which they lie. The numerical computations presented in Figure 13 show that the bifurcating non-symmetric 2-pulses lie on $z$-shaped branches; we believe that they can be predicted using the approach established in [2, Figure 1.7] for non-symmetric 1-pulses.

Other multi-pulses. The non-symmetric 2-pulses corresponding to the $z$-shaped branches shown in Figure 13 consist of two 1-pulses with roughly the same number of interior rolls. It is conceivable that non-symmetric 2- 

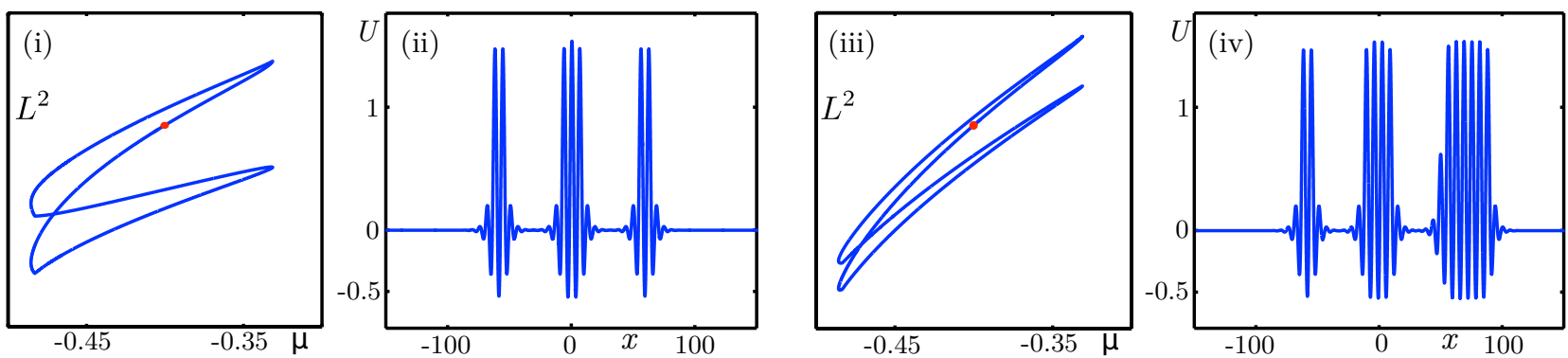

Figure 15: Panels (i) and (iii) contain solution branches of, respectively, symmetric and non-symmetric 3-pulses of (1.1). Sample profiles of symmetric and non-symmetric pulses along these branches are shown in panels (ii) and (iv), respectively.

pulses that consist of two 1-pulses with different numbers of interior rolls exist also. Indeed, Figure 14 contains such 2-pulses for (1.1), and we refer to [15] for a more comprehensive study of such pulses. All non-symmetric 2-pulses that we computed so far whose underlying 1-pulses have a different number of interior rolls lie on isolas. Using the smooth linearization around $u=0$ and following the approach we employed in $\S 3$, it is not difficult to derive the bifurcation equations that describe non-symmetric 2-pulses with transition times $L_{ \pm}$near the periodic orbit and separation distance $L_{0}$ near $u=0$. Preliminary calculations indicate that non-symmetric 2-pulses comprised of 1-pulses with different numbers of interior rolls always lie on isolas: Without more detailed knowledge about certain higher-order terms, however, it seems difficult to predict the actual shape of the isolas, and we leave this issue for future work. Finally, we expect that $N$-pulses with $N \geq 3$ behave in a similar fashion. Figure 15 contains computations of symmetric and non-symmetric 3-pulses of (1.1) that lie again on isolas, and we refer to [15] for additional computations.

Acknowledgments. Part of this work was carried out when Knobloch visited the UK in Autumn 2007: we are grateful to the London Mathematical Society for providing the funding for this visit. Sandstede was partially supported by a Royal Society-Wolfson Research Merit Award and by the NSF through grant DMS-0907904.

\section{References}

[1] D. Avitabile, D. J. B. Lloyd, J. Burke, E. Knobloch and B. Sandstede. To snake or not to snake in the planar Swift-Hohenberg equation. SIAM J. Appl. Dynam. Syst. 9 (2010) 704-733.

[2] M. Beck, J. Knobloch, D. J. B. Lloyd, B. Sandstede and T. Wagenknecht. Snakes, ladders, and isolas of localised patterns. SIAM J. Math. Anal. 41 (2009) 936-972.

[3] G. R. Belitskii and A. Y. Kopanskii. Sternberg theorem for equivariant Hamiltonian vector fields. Nonlinear Analysis 47 (2001) 4491-4499.

[4] J. Burke and E. Knobloch. Localized states in the generalized Swift-Hohenberg equation. Phys. Rev. E 73 (2006) 056211.

[5] J. Burke, S. M. Houghton and E. Knobloch. Swift-Hohenberg equation with broken reflection symmetry. Phys. Rev. E 80 (2009) 036202.

[6] J. Burke and E. Knobloch. Multipulse states in the Swift-Hohenberg equation. In: Dynamical Systems and Differential Equations (X. Hou, X. Lu, A. Miranville, J. Su and J. Zhu, eds.). Discr. Contin. Dynam. Syst. Suppl. (2009) 109-117. 
[7] A. R. Champneys, V. Kirk, E. Knobloch, B. E. Oldeman and J. D. M. Rademacher. Unfolding a tangent equilibrium-to-periodic heteroclinic cycle. SIAM J. Appl. Dyn. Syst. 8 (2009) 1261-1304.

[8] A. R. Champneys and J. F. Toland. Bifurcation of a plethora of multi-modal homoclinic orbits for autonomous Hamiltonian systems. Nonlinearity 6 (1993) 665-721.

[9] S. J. Chapman and G. Kozyreff. Exponential asymptotics of localised patterns and snaking bifurcation diagrams. Physica D 238 (2009) 319-354.

[10] P. Coullet, C. Riera and C. Tresser. Stable static localized structures in one dimension. Phys. Rev. Lett. 84 (2000) 3069-3072.

[11] J. H. P. Dawes. The emergence of a coherent structure for coherent structures: localized states in nonlinear systems. Phil. Trans. R. Soc. A 368 (2010) 3519-3534.

[12] R. L. Devaney. Homoclinic orbits in Hamiltonian systems. J. Differ. Eqns. 21 (1976) 431-438.

[13] J. Härterich. Cascades of reversible homoclinic orbits to a saddle-focus equilibrium. Physica D 112 (1998) $187-200$.

[14] G. H. M. van der Heijden, A. R. Champneys and J. M. T. Thompson. Spatially complex localisation in twisted elastic rods constrained to a cylinder. Int. J. Solids Struct. 39 (2002) 1863-1883.

[15] S. M. Houghton and T. Wagenknecht. Multi-pulses in the Swift-Hohenberg equation with broken symmetry. In preparation $(2010)$.

[16] E. Knobloch. Spatially localized structures in dissipative systems: open problems. Nonlinearity 21 (2008) T45-T60.

[17] J. Knobloch, T. Rieß and M. Vielitz. Nonreversible homoclinic snaking. In preparation (2010).

[18] G. Kozyreff and S. J. Chapman. Asymptotics of large bound states of localised structures. Phys. Rev. Lett. 97 (2006) 044502.

[19] L. M. Lerman. Dynamical phenomena near a saddle-focus homoclinic connection in a Hamiltonian system. J. Stat. Phys. 101 (2000) 357-372.

[20] J. Moser. On the generalisation of a theorem of A. Liapounoff. Comm. Pure Appl. Math. 11 (1958) $257-271$.

[21] B. E. Oldeman, A. R. Champneys and B. Krauskopf. Homoclinic branch switching: a numerical implementation of Lin's method. Int. J. Bif. Chaos 13 (2003) 2977-3000.

[22] B. Sandstede. Stability of multiple-pulse solutions. Trans. Amer. Math. Soc. 350 (1998) 429-472.

[23] B. Sandstede. Instability of localized buckling modes in a one-dimensional strut model. Phil. Trans. Roy. Soc. London A 355 (1997) 2083-2097.

[24] M. K. Wadee, C. D. Coman and A. P. Bassom. Solitary wave interaction phenomena in a strut buckling model incorporating restabilisation. Physica D 163 (2002) 26-48.

[25] P. D. Woods and A. R. Champneys. Heteroclinic tangles and homoclinic snaking in the unfolding of a degenerate reversible Hamiltonian Hopf bifurcation. Physica D 129 (1999) 147-170. 